\title{
Herschel/HIFI observations of red supergiants and yellow hypergiants
}

\section{Molecular inventory ${ }^{\star, \star \star, \star \star \star}$}

\author{
D. Teyssier ${ }^{1}$, G. Quintana-Lacaci ${ }^{2,5}$, A. P. Marston ${ }^{1}$, V. Bujarrabal ${ }^{3}$, J. Alcolea ${ }^{4}$, J. Cernicharo ${ }^{5}$, L. Decin ${ }^{6,7}$, \\ C. Dominik ${ }^{7,8}$, K. Justtanont ${ }^{9}$, A. de Koter ${ }^{7,10}$, G. Melnick ${ }^{11}$, K. M. Menten ${ }^{12}$, D. A. Neufeld ${ }^{13}$, H. Olofsson ${ }^{9,14}$, \\ P. Planesas ${ }^{4}$, M. Schmidt ${ }^{15}$, R. Soria-Ruiz ${ }^{4}$, F. L. Schöier ${ }^{9}{ }^{\dagger}$, R. Szczerba ${ }^{15}$, and L. B. F. M. Waters ${ }^{7,16}$ \\ ${ }^{1}$ European Space Astronomy Centre, Urb. Villafranca del Castillo, PO Box 50727, 28080 Madrid, Spain \\ e-mail: David.Teyssier@esa.int \\ 2 Instituto de Radio Astronomía Milimétrica (IRAM), Avenida Divina Pastora 7, Local 20, 18012 Granada, Spain \\ 3 Observatorio Astronómico Nacional (IGN), Ap 112, 28803 Alcalá de Henares, Spain \\ ${ }^{4}$ Observatorio Astronómico Nacional (IGN), Alfonso XII N³, 28014 Madrid, Spain \\ 5 CAB, INTA-CSIC, Ctra de Torrejón a Ajalvir, km 4, 28850 Torrejón de Ardoz, Madrid, Spain \\ ${ }^{6}$ Instituut voor Sterrenkunde, Katholieke Universiteit Leuven, Celestijnenlaan 200D, 3001 Leuven, Belgium \\ 7 Sterrenkundig Instituut Anton Pannekoek, University of Amsterdam, Science Park 904, 1098 Amsterdam, The Netherlands \\ 8 Department of Astrophysics/IMAPP, Radboud University Nijmegen, Nijmegen, The Netherlands \\ 9 Onsala Space Observatory, Dept. of Radio and Space Science, Chalmers University of Technology, 43992 Onsala, Sweden \\ 10 Astronomical Institute, Utrecht University, Princetonplein 5, 3584 CC Utrecht, The Netherlands \\ 11 Harvard-Smithsonian Center for Astrophysics, Cambridge, MA 02138, USA \\ 12 Max-Planck-Institut für Radioastronomie, Auf dem Hügel 69, 53121 Bonn, Germany \\ 13 The Johns Hopkins University, 3400 North Charles St, Baltimore, MD 21218, USA \\ 14 Department of Astronomy, AlbaNova University Center, Stockholm University, 10691 Stockholm, Sweden \\ 15 N. Copernicus Astronomical Center, Rabiańska 8, 87-100 Toruń, Poland \\ 16 SRON Netherlands Institute for Space Research, Sorbonnelaan 2, 3584 CA Utrecht, The Netherlands
}

Received 4 May 2012 / Accepted 26 July 2012

\begin{abstract}
Context. Red supergiant stars (RSGs) and yellow hypergiant stars (YHGs) are believed to be the high-mass counterparts of stars in the asymptotic giant branch (AGB) and early post-AGB phases. As such, they are scarcer and the properties and evolution of their envelopes are still poorly understood.

Aims. We study the mass-loss in the post main-sequence evolution of massive stars, through the properties of their envelopes in the intermediate and warm gas layers. These are the regions where the acceleration of the gas takes place and the most recent mass-loss episodes can be seen.

Methods. We used the HIFI instrument on-board the Herschel Space Observatory to observe sub-millimetre and far-infrared (FIR) transitions of CO, water, and their isotopologues in a sample of two RSGs (NML Cyg and Betelgeuse) and two YHGs (IRC+10420 and AFGL 2343) stars. We present an inventory of the detected lines and analyse the information revealed by their spectral profiles. A comparison of the line intensity and shape in various transitions is used to qualitatively derive a picture of the envelope physical structure. On the basis of the results presented in an earlier study, we model the $\mathrm{CO}$ and ${ }^{13} \mathrm{CO}$ emission in IRC+10420 and compare it to a set of lines ranging from the millimetre to the FIR.

Results. Red supergiants have stronger high-excitation lines than the YHGs, indicating that they harbour dense and hot inner shells contributing to these transitions. Consequently, these high- $J$ lines in RSGs originate from acceleration layers that have not yet reached the circumstellar terminal velocity and have narrower profiles than their flat-topped lower- $J$ counterparts. The YHGs tend to lack this inner component, in line with the picture of detached, hollow envelopes derived from studies at longer wavelengths. $\mathrm{NH}_{3}$ is only detected in two sources (NML Cyg and IRC+10420), which are also observed to be the strongest water-line emitters of the studied sample. In contrast, $\mathrm{OH}$ is detected in all sources and does not seem to correlate with the water line intensities. We show that the IRC+10420 model derived solely from millimetre low- $J$ CO transitions is capable of reproducing the high- $J$ transitions when the temperature in the inner shell is simply lowered by about $30 \%$.
\end{abstract}

Key words. stars: AGB and post-AGB - supergiants - circumstellar matter - submillimeter: stars

\footnotetext{
* Herschel is an ESA space observatory with science instruments provided by European-led Principal Investigator consortia and with important participation from NASA.

$\star \star$ Appendix A is available in electronic form at http://www . aanda.org
}

\footnotetext{
$\star \star \star$ FITS files of the spectra are only available at the CDS via anonymous ftp to cdsarc.u-strasbg.fr (130.79.128.5) or via http://cdsarc.u-strasbg.fr/viz-bin/qcat?J/A+A/545/A99

$\dagger$ Deceased 14 January 2011.
} 


\section{Introduction}

Red supergiant stars (RSGs) and yellow hypergiant stars (YHGs) are thought to be stages in the post main-sequence evolution of stars with initial masses between $\sim 10 M_{\odot}$ and $50 M_{\odot}$ (e.g. de Jager 1998; Meynet \& Maeder 2003; Levesque 2010). As such, RSGs and YHGs are the massive counterparts of AGB and (early) post-AGB stars. However, the very different properties of evolved massive stellar objects (RSGs, YHGs, Wolf-Rayet stars, luminous blue variable stars, Cepheid-like variables, supernovae etc.), particularly their distribution in the H-R diagram, is hard to interpret with a simple description of the evolution in this phase.

Both RSGs and YHGs are known to show complex massloss phenomena, which form circumstellar envelopes (CSEs) that can be very dense. Mass-loss plays an important role in the evolution of these objects (de Jager 1998; Meynet \& Maeder 2003): almost one half of their total initial mass can be ejected during these late phases, affecting in particular their possible later evolution into supernova events. Some objects have very high mass-loss rates, with episodic rates as high as $\dot{M} \sim$ $10^{-3} M_{\odot} \mathrm{yr}^{-1}$, while the circumstellar envelopes around others are very diffuse, corresponding to rates under $10^{-7} M_{\odot} \mathrm{yr}^{-1}$ (e.g. Castro-Carrizo et al. 2007, CC07 thereafter; Quintana-Lacaci 2008; Mauron \& Josselin 2011). From the theoretical point of view, very high rates are also expected, at least episodically, owing to a combination of high radiation pressure and atmospheric activity (e.g. Josselin \& Plez 2007; as happens in the AGB) or to the intrinsic instabilities characteristic of the yellow phases of the late evolution of high-mass stars (the so-called "yellow void"; see e.g. de Jager 1998).

Molecular lines have been one of the most powerful tools for the study of the properties of CSEs around AGB and postAGB stars. Similar data on RSGs and YHGs are rare, but in some cases molecular lines have yielded a quite comprehensive insight into their CSEs. For instance, using model fitting of mm- and submm-observations of several $\mathrm{CO}$ rotational lines in the RSG VY CMa, Decin et al. (2006) deduced mass-loss rates as high as $3 \times 10^{-4} M_{\odot} \mathrm{yr}^{-1}$, that varied on timescales of about 1000 yr. VY CMa is known to harbour a wide variety of molecular species, illustrating the very complex chemistry at play in its CSE (e.g. Tenenbaum et al. 2010, and references therein). Other less well-studied RSGs, such as VX Sgr and NML Cyg, also show high mass-loss rates of the order of $\sim 10^{-4} M_{\odot} \mathrm{yr}^{-1}$ (e.g. De Beck et al. 2010). An example of a low- $\dot{M}$ RSG is Betelgeuse ( $\alpha$ Ori), with a value of about $10^{-7}-10^{-6} M_{\odot} \mathrm{yr}^{-1}$, although this value is uncertain because its molecular emission may be weaker than in other objects owing to its molecular underabundance (see Huggins et al. 1994; Mauron \& Josselin 2011). The YHGs IRC+10420 and AFGL 2343 have also been well-studied in molecular emission. CC07 (see also Quintana-Lacaci et al. 2008) compared lineemission models with high-resolution maps of the CO $J=1-0$ and $J=2-1$ lines, deriving large $\dot{M}$ variations on timescales of about $1000 \mathrm{yr}$ and $\dot{M}$ maxima in excess of $10^{-3} M_{\odot} \mathrm{yr}^{-1}$. These objects also have many molecular lines and a very rich chemistry (Quintana-Lacaci et al. 2007). The total masses in the molecule-rich shells are particularly high for these two YHGs, $M_{\mathrm{tot}} \gtrsim 1 M_{\odot}$. The total masses of the molecular envelopes around RSGs are somewhat lower, between $0.1 M_{\odot}$ and $1 M_{\odot}$ for VY CMa, VX Sgr, and NML Cyg.

Despite the observational progress that has been made to date, the available data still fail to provide information on some basic parameters. Low- $J$ CO lines are good tracers of both the circumstellar mass distribution and kinematics, but these easily excited lines cannot probe warm gas with temperatures $T_{\mathrm{k}} \gtrsim 100 \mathrm{~K}$, which are expected to be present and sometimes dominant in these shells. The temperature itself is not well-determined under these conditions, which may affect the determination of the mass-loss rate and the total mass. To properly study these warm components, it is therefore necessary to observe lines in the far-infrared (FIR), involving level excitations comparable (in temperature units) to these moderate kinetic temperatures.

This paper presents Herschel/HIFI observations in the FIR of molecular lines from two RSGs, Betelgeuse and NML Cyg, and two YHGs, IRC+10420 and AFGL 2343. The observations are part of the Herschel guaranteed time key program HIFISTARS (PI V. Bujarrabal), devoted to the study of high-excitation molecular lines in (low- and high-mass) evolved stars. Companion observations of VY CMa, which are particularly rich and complex, will be discussed in another paper (Alcolea et al., in prep.). We present data of the $J=6-5, J=10-9$, and $J=16-15$ lines of ${ }^{12} \mathrm{CO}$ and ${ }^{13} \mathrm{CO}$, which are good probes of the excitation. We also discuss our observations of other molecules, including water vapour, $\mathrm{OH}$, and $\mathrm{NH}_{3}$, which are particularly useful to understanding more clearly the chemistry in these unusual objects.

\section{Observations and data processing}

The observations presented in this paper were obtained with the Heterodyne Instrument for the Far Infrared (HIFI; de Graauw et al. 2010), on-board the Herschel Space Observatory (Pilbratt et al. 2011). Similarly to other observations presented in the framework of the HIFISTARS key program (e.g. Bujarrabal et al. 2012), the main target lines of this project were collected through a handful of HIFI settings. Thanks to the instantaneous spectral coverage of 2.4-4 GHz offered by the Wide-Band Spectrometer (WBS), and since HIFI works with double-sideband (DSB) mixers, several other bonus lines could be covered simultaneously. A summary of the settings used and their main characteristics is given in Table 1 .

All data were taken in the double-beam switching (DBS) mode, consisting of chopping and nodding between the source line-of-sight and blank sky positions 3 arcmin on either sides of the source. This mode allows very fast switches between the sky positions, and therefore mitigates the detector drifts. Residual baseline ripples can however remain, especially in the HIFI bands 6 and 7 where the detectors used (hot electron bolometers, hereafter HEB) are more susceptible to instabilities.

We used the standard pipeline products that had been generated and made available at the Herschel Science Archive (HSA). These could be accessed via the HIPE software ${ }^{1}$. The HIFI pipeline brings all data onto a single-side-band $T_{\mathrm{A}}^{*}$ intensity scale. A side-band ratio of unity was assumed for all the lines reported in this paper. All individual spectra from the socalled levell were checked for outliers, and averaged for each of the two orthogonal mixer polarisations available on HIFI. In some rare cases, one of the polarisations was useless owing to an unpumped mixer, so its data had to be discarded. The sky positions observed by the two polarization mixers are not strictly the same, the misalignment varying between $\sim 6^{\prime \prime}$ in the lower bands to $1^{\prime \prime}$ in the higher bands (Roelfsema et al. 2012). Any such misalignment could in practice manifest itself (among other calibration effects) as intensity imbalance between the two

${ }^{1}$ HIPE is a joint development by the Herschel Science Ground Segment Consortium, consisting of ESA, the NASA Herschel Science Center, and the HIFI, PACS, and SPIRE consortia. 
Table 1. Summary of observational settings and telescope characteristics.

\begin{tabular}{lccccc}
\hline \hline $\begin{array}{l}\text { Setting } \\
\text { name }\end{array}$ & $\begin{array}{c}\text { LO freq. } \\
(\mathrm{GHz})\end{array}$ & $\begin{array}{c}\text { Typical } T_{\text {sys }} \\
(\mathrm{K}, \mathrm{DSB})\end{array}$ & Band & $\begin{array}{c}\text { Beam } \\
\text { size }\end{array}$ & $B_{\text {eff }}$ \\
\hline $14 \mathrm{~g}$ & 564.56 & 93 & $1 \mathrm{~B}$ & $37.5^{\prime \prime}$ & 0.754 \\
$12 \mathrm{f}$ & 653.55 & 131 & $2 \mathrm{~A}$ & $32.4^{\prime \prime}$ & 0.752 \\
17 & 686.42 & 142 & $2 \mathrm{~A}$ & $30.9^{\prime \prime}$ & 0.745 \\
$07 \mathrm{e}$ & 1106.90 & 416 & $4 \mathrm{~B}$ & $19.1^{\prime \prime}$ & 0.742 \\
$06 \mathrm{~d}$ & 1157.67 & 900 & $5 \mathrm{~A}$ & $18.3^{\prime \prime}$ & 0.639 \\
$05 \mathrm{c}$ & 1200.90 & 1015 & $5 \mathrm{~A}$ & $17.6^{\prime \prime}$ & 0.633 \\
$03 \mathrm{~b}$ & 1757.68 & 1580 & $7 \mathrm{~A}$ & $12.1^{\prime \prime}$ & 0.740 \\
16 & 1838.31 & 1300 & $7 \mathrm{~B}$ & $11.5^{\prime \prime}$ & 0.736 \\
\hline
\end{tabular}

Notes. The setting names are provided for cross-reference with other HIFISTARS results, and to ease the association of data with a given Herschel observation identifier (obsid). These obsid's are given in the figures of the appendix. For each setting, we indicate the corresponding HIFI band, the local oscillator frequency tuned for the observation and the system noise temperature achieved on average, together with the typical beam properties applying to each of them.

polarisations. We however averaged the two spectra whenever possible to improve the sensitivity. In practice, this implies that the effective beam size of the averaged data is slightly larger than the nominal spatial resolution.

A special treatment had to be applied to some of the data taken in the HEB bands owing to the standing wave affecting in particular these detectors. This spectral artefact is not optical in nature, hence cannot be optimally treated by applying the standard defringing methods to sine-wave baseline distortion. A novel method is currently being developed to perform an alternative pipeline correction of these features (Higgins 2011), which may, however, be ineffective when the line widths become similar to those of the artefact structure. Because this particular situation applies to the sources treated in this study, we decided not to adopt this approach. We developed instead a semi-automated procedure that discards individual spectra where the contribution of the baseline ripple is significantly stronger than the expected radiometric noise. This results in the rejection of up to half of the non-averaged spectra, and therefore increases the noise in the final data, in spite of the more reliable baseline.

Finally, all spectra were converted onto a $T_{\mathrm{mb}}$ scale, by applying the main beam efficiencies reported by the HIFI Instrument Control Centre, which are reproduced in Table 1. We based our absolute-calibration accuracy estimates on the error budget reported by Roelfsema et al. (2012). On top of this, we considered an additional contribution arising from the standing wave described above for bands 6 and 7 . Adding up all error contributions in quadrature only provided lower limits to the typical calibration errors. In practice, the calibration error that we assumed in this study is $15 \%$ in bands 1 and 2, 20\% in bands 3 to 5, and 30\% in bands 6 and 7. The collection of all full-band spectra is compiled in Appendix A.

\section{Results}

Table 2 summarises the main stellar parameters of the sample studied here. In the following sub-sections, we give an overall inventory of all probed and detected species, together with the extracted line characteristics in the form of the line peak and integrated intensities (see Tables 3 and 4 for a compilation of these results). The high spectral resolution of HIFI also allows us to distinguish and discuss the contribution of particular shell layers to the emission of the respective species.

\subsection{NML Cyg}

NML Cyg is a red supergiant of spectral type M6. It has an intermediate mass-loss rate of order $8.7 \times 10^{-5} M_{\odot} \mathrm{yr}^{-1}$ (De Beck et al. 2010).

NML Cyg exhibits the richest spectrum of all four sources presented here, although, among the full sample of RSGs, it is largely surpassed by the line density of VY CMa (Alcolea et al., in prep.). Apart from this latter, it is also the source with the strongest line emission when considering species and transitions individually (see Tables 3 and 4, and Fig. 9). While the detection of water vapour lines in other sources is mostly limited to the ground-state transitions of both ortho- and para- $\mathrm{H}_{2} \mathrm{O}$, NML Cyg shows transitions with energy levels in excess of $2000 \mathrm{~K}$. One noticeable detection is that of the $\mathrm{H}_{2} \mathrm{O}$ maser line at $658 \mathrm{GHz}$. The measured flux of $400 \mathrm{Jy}$ agrees well with the first detection of this line by Menten \& Young (1995), indicating that there is no evidence of large variability. Water maser emission at $22 \mathrm{GHz}$ has also been reported in this source (e.g. Nagayama et al. 2008). While the velocity distribution of this latter exhibits two shifted components at $-25 \mathrm{~km} \mathrm{~s}^{-1}$ and $+5 \mathrm{~km} \mathrm{~s}^{-1}$ respectively, the $658 \mathrm{GHz}$ maser is peaked at the star velocity of $v_{\text {LSR }}=0 \mathrm{~km} \mathrm{~s}^{-1}$, with a relatively narrow profile compared to the other submm transitions, and a blue-shifted shoulder. This is in contrast to the rest of the water vapour lines, where the blue side is usually narrower than the red side. The narrower line width shows that the maser originates from inner layers still in acceleration.

NML Cyg also displays a uniquely rich spectrum of SO, rotational transitions from the ground, and vibrationally excited states of $\mathrm{SiO}$, as well as isotopes of the $\mathrm{SiO}$, though only from the ground vibrational state (see Fig. 3). The detection of high- $J$ transitions of $\mathrm{SiO}$ confirms the presence of warm and dense gas at the centre of the envelope. This is a noticeable observation since, as shown in the following sections, the other sources seem to have rather hollow envelopes. This result is corroborated by the presence of very high excitation $\mathrm{H}_{2} \mathrm{O}$ transitions, and tells us that the mass-loss rate is probably still very high today. Finally, NML Cyg is one of the few sources showing a strong emission in $\mathrm{NH}_{3}$, and the only RSG together with VY CMa to exhibit detectable emission from other hydrides such as $\mathrm{HCN}$ and $\mathrm{H}_{2} \mathrm{~S}$.

The velocity structure of the various species and transitions displays a great variety of profile shapes. As is well-known, the line shape essentially depends on the line opacity, but also on the velocity profile of the layers where the line is emitting. Typically, the transition from a rectangular (flat-top) to a parabolic profile reflects the effect of optical depth in a constant velocity flow, while the transition to a triangular, narrower profile comes about when one probes the inner and warmer acceleration region with higher excitation lines. A recent study of submm molecular lines of $\mathrm{CO}$ and $\mathrm{H}_{2} \mathrm{O}$ in O-rich AGB stars (Justtanont et al. 2012) indicates that the line width tends to be smaller for high excitation lines formed closer to the star, pointing to an accelerated outflow.

In NMLCyg, this kind of transition is particularly pronounced when looking at the evolution between the $J=6-5$, $J=10-9$, and $J=16-15$ transitions of ${ }^{12} \mathrm{CO}$ and ${ }^{13} \mathrm{CO}$, respectively (see Fig. 1). That the same trend is observed in both optically thick and thin isotopes suggests that this is not primarily an opacity effect and we indeed detect the velocity gradient in the innermost layers of the envelope. The same behaviour is observed for increasingly higher energy levels of the $p-\mathrm{H}_{2} \mathrm{O}$ and 
Table 2. Observed coordinates and additional stellar parameters for the two RSGs and the two YHGs considered in this study: spectral type, distance and luminosity.

\begin{tabular}{|c|c|c|c|c|c|c|}
\hline Source & $\alpha(\mathrm{J} 2000)$ & $\delta(\mathrm{J} 2000)$ & Spectral type & Distance (pc) & Luminosity $\left(L_{\odot}\right)$ & References \\
\hline \multicolumn{7}{|c|}{ Red supergiants } \\
\hline Betelgeuse & 055510.3 & +072425.4 & M2 & 150 & $4 \times 10^{5}$ & 1 \\
\hline NML Cyg & 204625.5 & +400659.6 & M6 & 1700 & $6 \times 10^{5}$ & 2 \\
\hline \multicolumn{7}{|c|}{ Yellow hypergiants } \\
\hline IRC +10420 & 192648.0 & +112116.7 & F8 & 5000 & $7 \times 10^{5}$ & 3 \\
\hline AFGL 2343 & 191358.6 & +000731.9 & G5 & 6000 & $6 \times 10^{5}$ & 4 \\
\hline
\end{tabular}

References. 1) Mauron \& Josselin (2011); 2) Schuster et al. (2009); 3) de Jager (1998); 4) Hawkins et al. (1995); Reddy \& Hrivnak (1999).

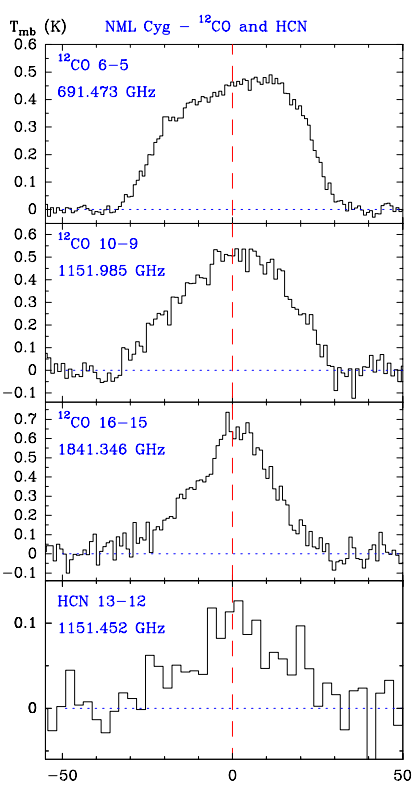

Velocity $(\mathrm{km} / \mathrm{s})$
Velocity $(\mathrm{km} / \mathrm{s})$

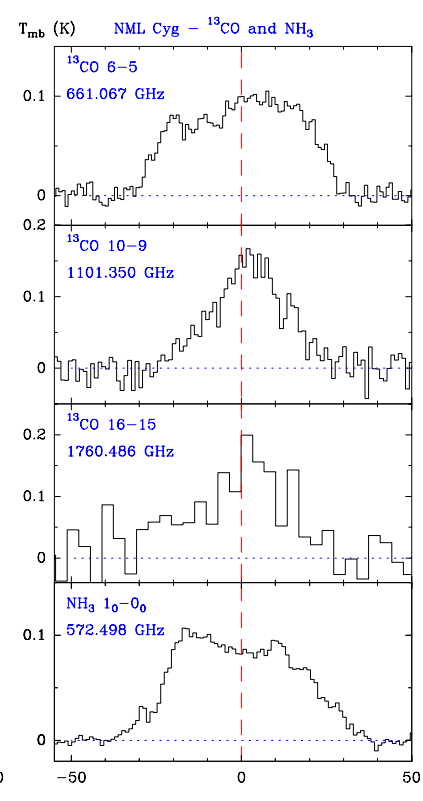

Fig. 1. Individual line spectra collected with Herschel/HIFI on NML Cyg for $\mathrm{CO},{ }^{13} \mathrm{CO}, \mathrm{NH}_{3}$, and $\mathrm{HCN}$. The dashed line indicates the source systemic velocity.

$p-\mathrm{H}_{2}^{18} \mathrm{O}$ transitions (see Fig. 2). Opacity, however, also plays a role in the line shape, as can be seen from the narrowing of the line profile between the respective $J=10-9$ transitions of ${ }^{12} \mathrm{CO}$ and ${ }^{13} \mathrm{CO}$, which have similar excitation temperatures. Interestingly, the $1834 \mathrm{GHz} \mathrm{OH}$ line profile closely resembles that of the mid- $J \mathrm{CO}$ transitions, suggesting that its emission arises predominantly in the outer layers where the terminal velocity has been reached.

Another peculiarity of the velocity structure can be observed in the $\mathrm{NH}_{3}$ line profile (see Fig. 1). Unlike the other lines studied, that exhibit an overall single-peaked profile, the $\mathrm{NH}_{3}$ emission has two separate peaks on either side of the systemic velocity. In particular, this structure provides evidence for a component lying at velocity around $-18 \mathrm{~km} \mathrm{~s}^{-1}$. We note that this blue-shifted velocity component coincides with a prominent contribution of the $v=1, J=2-1, \mathrm{SiO}$ maser emission at $43 \mathrm{GHz}$ (Boboltz \& Marvel 2000), showing up at sub-arcsec scales as a marked bipolar structure also reported in mid-IR maps of the dust emission (e.g. Monnier et al. 2004). Menten et al. (2010) already emphasized the peculiar behaviour of ammonia in other giant stars such as VY CMa or IRC+10420, reporting unexpectedly high abundances in their envelope. We speculate that the bulk of the $\mathrm{NH}_{3}$

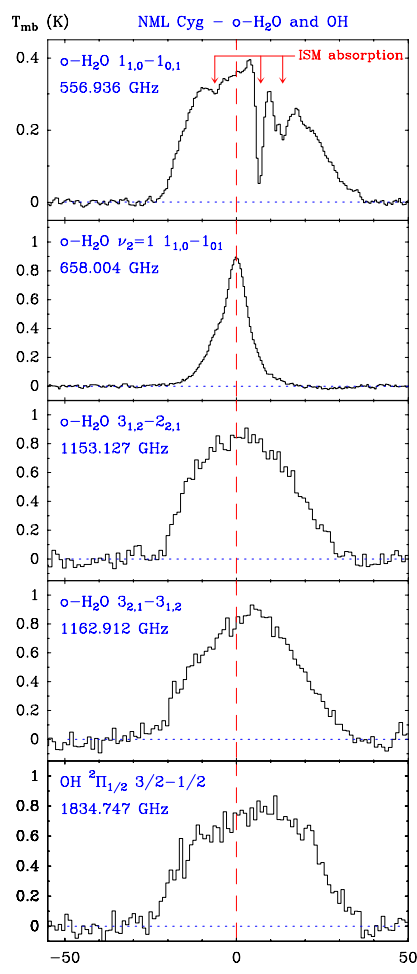

Velocity $(\mathrm{km} / \mathrm{s})$

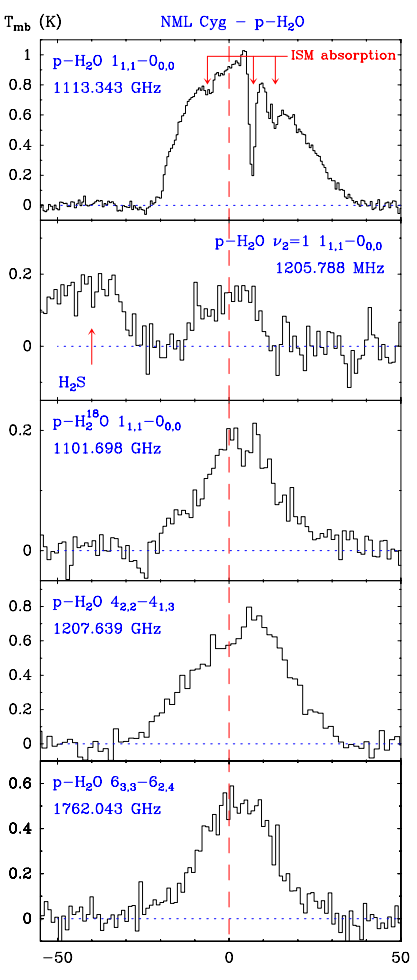

Velocity $(\mathrm{km} / \mathrm{s})$
Fig. 2. Same as Fig. 1 for $\mathrm{OH}, \mathrm{H}_{2} \mathrm{O}$ and isotopologues in NML Cyg

abundance could actually be quite widely distributed over the circumstellar shell.

Finally, we note the various ISM absorption signatures seen only in the two ground-state transitions of $o-\mathrm{H}_{2} \mathrm{O}$ and $p-\mathrm{H}_{2} \mathrm{O}$. While these features were also observed in low- $J$ CO transitions (Kemper et al. 2003), they are not present in the other transitions probed by Herschel, confirming that they arise from either a diffuse or cold gas component. We also note that there is a narrow emission component in the ground state $o-\mathrm{H}_{2} \mathrm{O}$ and $p-\mathrm{H}_{2} \mathrm{O}$ at $5 \mathrm{~km} \mathrm{~s}^{-1}$. This small peak was also observed in the ${ }^{12} \mathrm{CO} J=2-1$ line of Kemper et al. (2003) and probably originates from a small ISM clump along the line-of-sight. This interpretation is supported by recent distance measurements of NML Cyg (Zhang et al. 2012) and foreground interstellar gas (Rygl et al. 2012). 
D. Teyssier et al.: Herschel/HIFI observations of red supergiants and yellow hypergiants. I.

Table 3. Spectral line results for NML Cyg and IRC+10420.

\begin{tabular}{|c|c|c|c|c|c|c|c|c|c|}
\hline Species & Transition & $\begin{array}{c}E_{\text {upp }}^{\dagger} \\
(\mathrm{K})\end{array}$ & $\begin{array}{c}\text { Rest freq. } \\
(\mathrm{GHz})\end{array}$ & $\begin{array}{r}\operatorname{Peak}^{(7)} \\
(\mathrm{mK})\end{array}$ & $\begin{array}{c}\text { NML Cyg } \\
\text { Integ. intensity } \\
\left(\mathrm{K} \mathrm{km} \mathrm{s}^{-1}\right)\end{array}$ & $\begin{array}{l}\text { Vel. range }{ }^{(2)} \\
\text { LSR }\left(\mathrm{km} \mathrm{s}^{-1}\right)\end{array}$ & $\begin{array}{c}\operatorname{Peak}^{(7)} \\
(\mathrm{mK})\end{array}$ & $\begin{array}{c}\text { IRC }+10420 \\
\text { Integ. intensity } \\
\left(\mathrm{K} \mathrm{km} \mathrm{s}^{-1}\right)\end{array}$ & $\begin{array}{l}\text { Vel. range }{ }^{(2)} \\
\text { LSR }\left(\mathrm{km} \mathrm{s}^{-1}\right)\end{array}$ \\
\hline${ }^{12} \mathrm{CO}$ & $\begin{array}{c}J=6-5 \\
J=10-9 \\
J=16-15\end{array}$ & $\begin{array}{l}116 \\
304 \\
752\end{array}$ & $\begin{array}{c}691.473 \\
1151.985 \\
1841.346\end{array}$ & $\begin{array}{l}471(13)^{(4)} \\
479(39)^{(4)} \\
666(47)^{(4)}\end{array}$ & $\begin{array}{l}19.6 \\
19.1 \\
17.6\end{array}$ & $\begin{array}{l}-34 ; 33] \\
{[-33 ; 30]} \\
{[-38 ; 28]}\end{array}$ & $\begin{array}{l}364(20)^{(3)} \\
243(44)^{(4)} \\
133(44)^{(5)}\end{array}$ & $\begin{array}{l}19.1 \\
14.7 \\
6.57\end{array}$ & $\begin{array}{l}37 ; 115] \\
{[36 ; 114]} \\
{[38 ; 111]}\end{array}$ \\
\hline${ }^{13} \mathrm{CO}$ & $\begin{array}{c}J=6-5 \\
J=10-9 \\
J=16-15\end{array}$ & $\begin{array}{l}111 \\
291 \\
719\end{array}$ & $\begin{array}{c}661.067 \\
1101.350 \\
1760.486\end{array}$ & $\begin{array}{c}95(7)^{(4)} \\
141(17)^{(4)} \\
135(43)^{(6)}\end{array}$ & $\begin{array}{l}4.06 \\
3.80 \\
4.75\end{array}$ & $\begin{array}{l}{[-34 ; 33]} \\
{[-25 ; 31]} \\
{[-31 ; 27]}\end{array}$ & $\begin{array}{l}57(6)^{(4)} \\
60(20)^{(4)} \\
<138^{(1,5)}\end{array}$ & $\begin{array}{c}2.79 \\
2.8 \\
-\end{array}$ & $\begin{array}{c}{[36 ; 113]} \\
{[43 ; 107]} \\
-\end{array}$ \\
\hline$o-\mathrm{H}_{2} \mathrm{O}$ & $\begin{array}{l}J_{K_{\mathrm{a}}, K_{\mathrm{c}}}=1_{1,0}-1_{0,1} \\
J_{K_{\mathrm{a}}, K_{\mathrm{c}}}=3_{1,2}-2_{2,1} \\
J_{K_{\mathrm{a}}, K_{\mathrm{c}}}=3_{2,1}-3_{1,2}\end{array}$ & $\begin{array}{c}27 \\
215 \\
271\end{array}$ & $\begin{array}{c}556.936 \\
1153.127 \\
1162.912\end{array}$ & $\begin{array}{c}386(6)^{(3)} \\
848(41)^{(4)} \\
869(42)^{(4)}\end{array}$ & $\begin{array}{l}12.2 \\
28.8 \\
29.7\end{array}$ & $\begin{array}{l}{[-23 ; 37]} \\
{[-23 ; 35]} \\
{[-31 ; 34]}\end{array}$ & $\begin{array}{c}168(6)^{(3)} \\
333(42)^{(4)} \\
195(41)^{(4)}\end{array}$ & $\begin{array}{l}6.80 \\
13.7 \\
8.41\end{array}$ & $\begin{array}{l}{[43 ; 118]} \\
{[38 ; 111]} \\
{[40 ; 112]}\end{array}$ \\
\hline$p-\mathrm{H}_{2} \mathrm{O}$ & $\begin{array}{l}J_{K_{\mathrm{a}}, K_{\mathrm{c}}}=1_{1,1}-0_{0,0} \\
J_{K_{\mathrm{a}}, K_{\mathrm{c}}}=4_{2,2}-4_{1,3} \\
J_{K_{\mathrm{a}}, K_{\mathrm{c}}}=6_{3,3}-6_{2,4}\end{array}$ & $\begin{array}{c}53 \\
454 \\
952\end{array}$ & $\begin{array}{l}1113.343 \\
1207.639 \\
1762.043\end{array}$ & $\begin{array}{l}993(24)^{(3)} \\
726(47)^{(5)} \\
526(43)^{(4)}\end{array}$ & $\begin{array}{l}30.8 \\
22.9 \\
14.0\end{array}$ & $\begin{array}{l}{[-23 ; 38]} \\
{[-32 ; 34]} \\
{[-29 ; 30]}\end{array}$ & $\begin{array}{l}329(21)^{(3)} \\
<144^{(1,4)} \\
<180^{(1,4)}\end{array}$ & $\begin{array}{c}12.7 \\
- \\
-\end{array}$ & $\begin{array}{c}{[46 ; 115]} \\
- \\
-\end{array}$ \\
\hline$o-\mathrm{H}_{2} \mathrm{O} v_{2}=1$ & $J_{K_{\mathrm{a}}, K_{\mathrm{c}}}=1_{1,0}-1_{0,1}$ & 2326 & 658.004 & $882(9)^{(3)}$ & 9.53 & {$[-26 ; 18]$} & $<15^{(1,5)}$ & - & - \\
\hline$p-\mathrm{H}_{2} \mathrm{O} v_{2}=1$ & $J_{K_{\mathrm{a}}, K_{\mathrm{c}}}=1_{1,1}-0_{0,0}$ & 2352 & 1205.788 & $112(44)^{(4)}$ & 2.64 & {$[-18 ; 17]$} & $<144^{(1,4)}$ & - & - \\
\hline$p-\mathrm{H}_{2}^{18} \mathrm{O}$ & $J_{K_{\mathrm{a}}, K_{\mathrm{c}}}=1_{1,1}-0_{0,0}$ & 53 & 1101.698 & $185(18)^{(4)}$ & 5.05 & {$[-24 ; 37]$} & $<60^{(1,4)}$ & - & - \\
\hline $\mathrm{SiO}$ & $J=16-15$ & 283 & 694.294 & $118(14)^{(4)}$ & 3.42 & {$[-32 ; 30]$} & $<35^{(1,5)}$ & - & - \\
\hline $\mathrm{SiO} v=1$ & $\begin{array}{l}J=13-12 \\
J=15-14\end{array}$ & $\begin{array}{l}1957 \\
2017\end{array}$ & $\begin{array}{l}560.325 \\
646.429\end{array}$ & $\begin{array}{l}17(3)^{(5)} \\
30(5)^{(5)}\end{array}$ & $\begin{array}{l}0.39 \\
0.50\end{array}$ & $\begin{array}{l}{[-25 ; 27]} \\
{[-25 ; 25]}\end{array}$ & $\begin{array}{l}<15^{(1,4)} \\
<15^{(1,5)}\end{array}$ & - & $\begin{array}{l}- \\
-\end{array}$ \\
\hline${ }^{29} \mathrm{SiO}$ & $\begin{array}{l}J=13-12 \\
J=26-25\end{array}$ & $\begin{array}{l}187 \\
722\end{array}$ & $\begin{array}{c}557.179 \\
1112.798\end{array}$ & $\begin{array}{c}30(4)^{(5)} \\
50(17)^{(5)}\end{array}$ & $\begin{array}{l}0.95 \\
1.10\end{array}$ & $\begin{array}{l}{[-25 ; 26]} \\
{[-21 ; 13]}\end{array}$ & $\begin{array}{l}<15^{(1,4)} \\
<60^{(1,4)}\end{array}$ & - & $\begin{array}{l}- \\
-\end{array}$ \\
\hline${ }^{30} \mathrm{SiO}$ & $J=26-25$ & 713 & 1099.708 & $59(14)^{(5)}$ & 0.77 & {$[-17 ; 24]$} & $<60^{(1,4)}$ & - & - \\
\hline SO & $\begin{array}{l}J_{K}=13_{14}-12_{13} \\
J_{K}=13_{12}-12_{11} \\
J_{K}=13_{13}-12_{12} \\
J_{K}=15_{16}-14_{15}\end{array}$ & $\begin{array}{l}193 \\
194 \\
201 \\
253\end{array}$ & $\begin{array}{l}560.178 \\
558.087 \\
559.320 \\
645.875\end{array}$ & $\begin{array}{l}13(4)^{(5)} \\
13(4)^{(5)} \\
20(3)^{(5)} \\
21(4)^{(5)}\end{array}$ & $\begin{array}{l}0.40 \\
0.56 \\
0.49 \\
0.54\end{array}$ & $\begin{array}{l}{[-22 ; 23]} \\
{[-35 ; 29]} \\
{[-22 ; 25]} \\
{[-21 ; 22]}\end{array}$ & $\begin{array}{l}<15^{(1,4)} \\
<15^{(1,4)} \\
<15^{(1,4)} \\
<15^{(1,5)}\end{array}$ & $\begin{array}{l}- \\
- \\
- \\
-\end{array}$ & $\begin{array}{l}- \\
- \\
- \\
-\end{array}$ \\
\hline $\mathrm{H}_{2} \mathrm{~S}$ & $J_{K_{\mathrm{a}}, K_{\mathrm{c}}}=3_{1,2}-2_{2,1}$ & 137 & 1196.012 & $149(33)^{(4)}$ & 3.83 & {$[-13 ; 22]$} & $<144^{(1,4)}$ & - & - \\
\hline $\mathrm{HCN}$ & $J=13-12$ & 386 & 1151.452 & $86(27)^{(6)}$ & 3.48 & {$[-35 ; 32]$} & $<126^{(1,4)}$ & - & - \\
\hline $\mathrm{H}^{13} \mathrm{CN}$ & $J=8-7$ & 147 & 690.552 & $<18^{(1,6)}$ & - & - & $<35^{(1,5)}$ & - & - \\
\hline $\mathrm{NH}_{3}$ & $J_{K}=1_{0}-0_{0}$ & 27 & 572.498 & $102(3)^{(4)}$ & 4.08 & {$[-38 ; 38]$} & $93(5)^{(4)}$ & 2.93 & {$[41 ; 116]$} \\
\hline $\mathrm{OH}^{2} \Pi_{1 / 2}$ & $J=3 / 2-1 / 2$ & 270 & 1834.747 & $800(46)^{(4)}$ & 31.4 & {$[-25 ; 36]$} & $599(58)^{(4)}$ & 33.5 & {$[37 ; 113]$} \\
\hline
\end{tabular}

Notes. For each line we indicate the corresponding upper energy level and rest frequency, together with the measured line peak intensity, line integrated intensity, and the velocity range used to compute this latter. ${ }^{(\dagger)}$ For $o-\mathrm{H}_{2} \mathrm{O}$, the energies are relative to that of the lowest ortho level $\left(J_{K_{\mathrm{a}}, K_{\mathrm{c}}}=1_{0,1}\right)$, since $o-\mathrm{H}_{2} \mathrm{O}$ and $p-\mathrm{H}_{2} \mathrm{O}$ behave as different species. ${ }^{(1)}$ Non-detections are given as 3- $\sigma$; ${ }^{(2)}$ range over which the integrated intensity

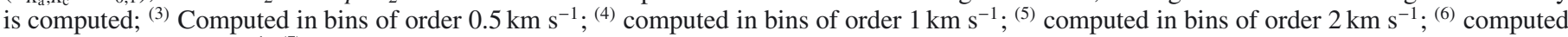
in bins of order $3 \mathrm{~km} \mathrm{~s}^{-1}$; ${ }^{(7)}$ computed as the line maximum minus $1.5 \times$ the noise rms, indicated in italics between brackets $(1-\sigma)$.

\subsection{Betelgeuse}

Betelgeuse ( $\alpha$ Ori) is a red supergiant of spectral type M2. It has a relatively low mass-loss rate of order $2 \times 10^{-7} M_{\odot} \mathrm{yr}^{-1}$ (De Beck et al. 2010).

Betelgeuse appears as a source that is particularly strong in ${ }^{12} \mathrm{CO}$ and ${ }^{13} \mathrm{CO}$ emission. In absolute intensities, it is the brightest of all targets reported here for these molecules. In contrast, it exhibits faint $\mathrm{H}_{2} \mathrm{O}$ and $\mathrm{OH}$ lines, especially in relation to the CO line intensity (Fig. 4, see also Fig. 9). Betelgeuse has a particularly small distance, $150 \mathrm{pc}$, which, despite its low mass-loss rate, explains its high $\mathrm{CO}$ line intensities. Owing to this weak mass loss, the envelope density is lower, and the weak water emission could be a consequence of enhanced photodissociation by stellar and interstellar ultra-violet (UV) photons as $\mathrm{H}_{2} \mathrm{O}$ is more easily photodissociated than $\mathrm{CO}$ (e.g. Justtanont et al. 1999). We recall that this source has very strong finestructure lines of both neutral atoms and low-energy ions (e.g. 
Table 4. Same as Table 3 for the spectral line observed in AFGL 2343 and Betelgeuse.

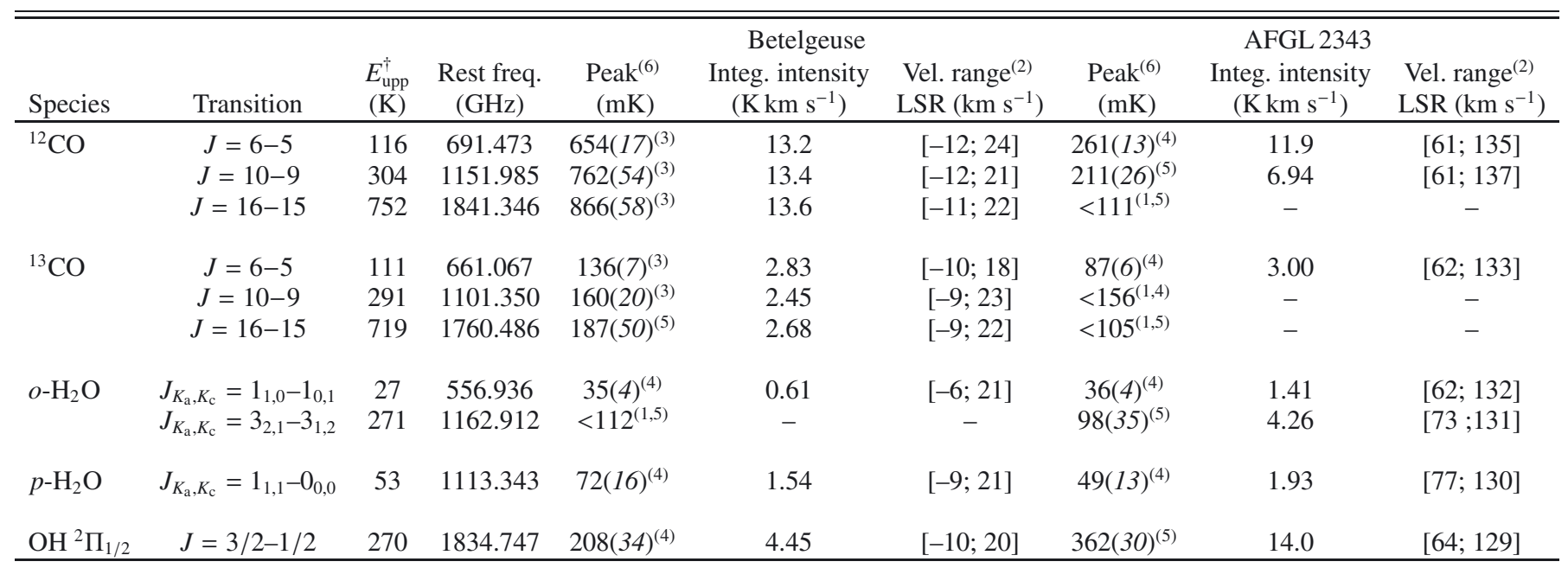

Notes. ${ }^{(\dagger)}$ For $o-\mathrm{H}_{2} \mathrm{O}$, the energies are relative to that of the lowest ortho level $\left(J_{K_{\mathrm{a}}, K_{\mathrm{c}}}=1_{0,1}\right)$, since $o-\mathrm{H}_{2} \mathrm{O}$ and $p$ - $\mathrm{H}_{2} \mathrm{O}$ behave as different species. (1) Non-detections are given as 3- $\sigma$; ${ }^{(2)}$ range over which the integrated intensity is computed; ${ }^{(3)}$ computed in bins of order $0.5 \mathrm{~km} \mathrm{~s}{ }^{-1}$; ${ }^{(4)}$ computed in bins of order $1 \mathrm{~km} \mathrm{~s}^{-1}$; ${ }^{(5)}$ computed in bins of order $2 \mathrm{~km} \mathrm{~s}^{-1}$; ${ }^{(6)}$ computed as the line maximum minus $1.5 \times$ the noise rms, indicated in italics between brackets $(1-\sigma)$.

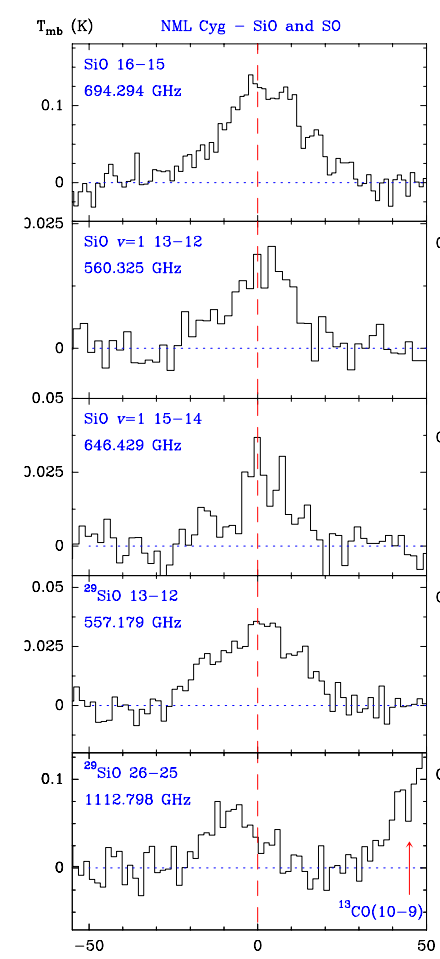

Velocity $(\mathrm{km} / \mathrm{s})$

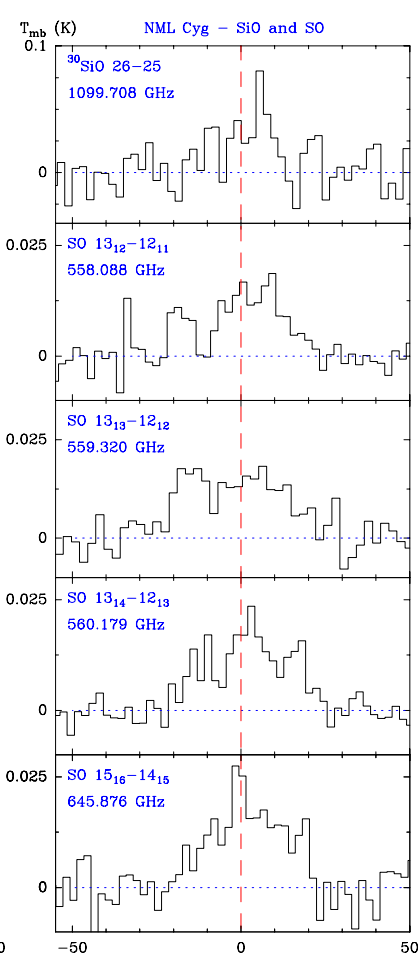

Velocity $(\mathrm{km} / \mathrm{s})$

Fig. 3. Same as Fig. 2 for $\mathrm{SiO},{ }^{29} \mathrm{SiO},{ }^{30} \mathrm{SiO}$ and $\mathrm{SO}$ in NML Cyg.

Haas \& Glassgold 1993; Castro-Carrizo et al. 2001), which are thought to be abundant in the inner envelope owing to the UV emission of the extended chromosphere of the star.

As in NML Cyg, the ${ }^{12} \mathrm{CO}$ and ${ }^{13} \mathrm{CO}$ line profiles become narrower as the excitation temperature increases, which again suggests that the high-transition lines form predominantly in the acceleration region. Betelgeuse is thought to have a particularly hot inner circumstellar region (Rodgers \& Glassgold 1991), with temperatures higher than about $1000 \mathrm{~K}$ out to $10^{15} \mathrm{~cm}$ from the star (i.e. occupying about $1^{\prime \prime}$ ). The evolution in the line profile

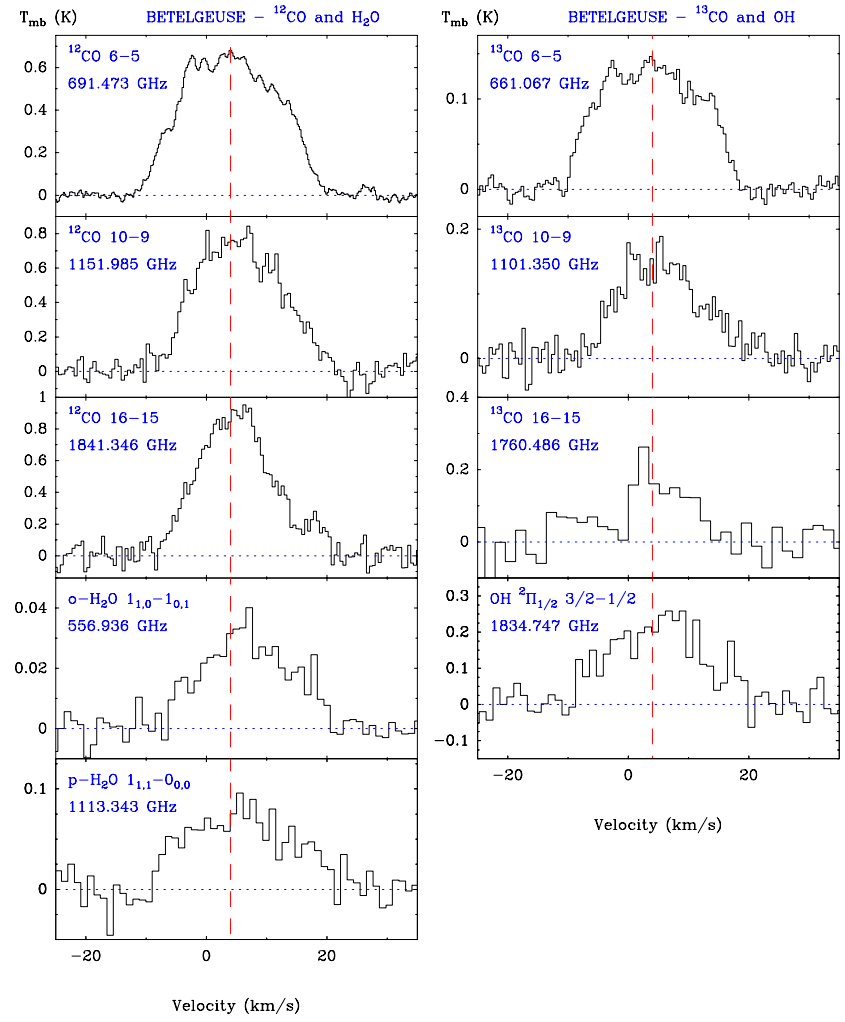

Fig. 4. Same as Fig. 2 for lines detected in Betelgeuse.

can be clearly seen if one compares the spectra reported here to the nearly flat-top shape of the ${ }^{12} \mathrm{CO} J=3-2$ spectrum observed by Kemper et al. (2003). There are also some small spectral components in our CO profiles sitting on top of the overall parabolic or triangular profiles. Those small structures could be related to clumps in the inner envelope, which have been reported in mid-IR observations of the inner $3^{\prime \prime}$ of the nebula (Kervella et al. 2011). 
D. Teyssier et al.: Herschel/HIFI observations of red supergiants and yellow hypergiants. I.
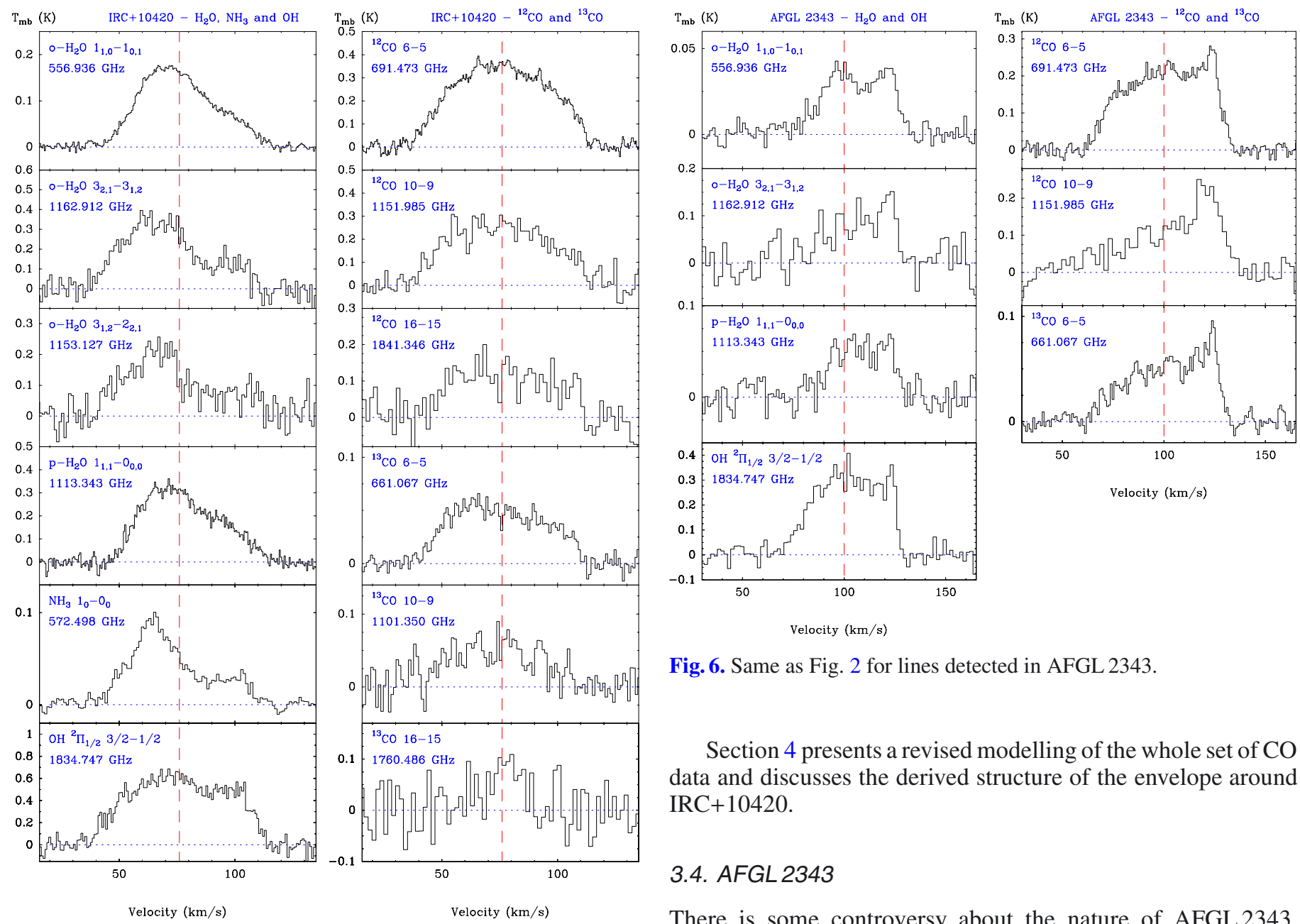

Fig. 6. Same as Fig. 2 for lines detected in AFGL 2343.

Section 4 presents a revised modelling of the whole set of $\mathrm{CO}$ data and discusses the derived structure of the envelope around IRC +10420 .

\subsection{AFGL 2343}

Fig. 5. Same as Fig. 2 for lines detected in IRC+10420.

\section{3. $I R C+10420$}

IRC +10420 is a yellow hypergiant, of spectral type F8. It has a strongly variable mass-loss rate, with highest values around $3 \times$ $10^{-4} M_{\odot} \mathrm{yr}^{-1}(\mathrm{CC} 07)$.

IRC +10420 presents the second richest species inventory after NMLCyg (Fig. 5), although it features none of the SO, $\mathrm{SiO}$, or C-bearing molecules detected there (other than $\mathrm{CO}$ and ${ }^{13} \mathrm{CO}$ ). Noticeably, strong lines of $\mathrm{NH}_{3}$ and $\mathrm{OH}$ are observed, where hydroxil is the brightest of all detected lines in this source. Only a handful of water lines are detected on top of the fundamental levels of $o-\mathrm{H}_{2} \mathrm{O}$ and $p-\mathrm{H}_{2} \mathrm{O}$ (see Table 3). While the CO lines have roughly parabolic line profiles, the rest of the detected species display a strong asymmetry in the form of a blue-shifted intensity excess. This feature is particularly clear in the $\mathrm{H}_{2} \mathrm{O}$ and $\mathrm{NH}_{3}$ profiles (Fig. 5, see also Menten et al. 2010), but only mildly observed in $\mathrm{OH}$. A similar trend was already reported in mm-wavelengths transitions of e.g. $\mathrm{CS}, \mathrm{H}^{13} \mathrm{CN}$, or ${ }^{29} \mathrm{SiO}$ by Quintana-Lacaci et al. (2007). CC07 demonstrated that the main contribution of this spectral component arises from a region in the western halo of the envelope (see their Fig. 8). This isolated shell component was also reported in high-resolution mid-IR maps by Lagadec et al. (2011). We propose that this spectral feature is due to an enhanced abundance of certain species in these layers, as the model presented by $\mathrm{CC} 07$ predicts only moderate $(\sim 50 \mathrm{~K})$ temperatures at this radius. $\mathrm{H}_{2} \mathrm{O}$ and $\mathrm{NH}_{3}$ are often believed to appear as a result of shock-induced chemistry, suggesting that this gas layer could be composed of shocked material.

There is some controversy about the nature of AFGL 2343, which has been proposed by certain authors to be a post-AGB (low-mass) object (see Ferguson \& Ueta 2010, and references therein), located at a relatively short distance $(\sim 1 \mathrm{kpc}$, instead of $\sim 6 \mathrm{kpc}$ if it is a luminous hypergiant; note that no reliable parallax measurement of this source exists). The majority of published papers favour the massive-star hypothesis, in particular those reporting studies of the circumstellar shell around AFGL 2343 (Reddy \& Hrivnak 1999; Gledhill et al. 2001; CC07, Quintana-Lacaci et al. 2008), since the shell seems very extended and similar to that of the well-studied IRC +10420 . The fast and mostly isotropical expansion of its envelope also supports the YHG nature of AGL 2343. Accordingly, we also assume that AFGL 2343 is a YHG, of spectral type G5. CC07 reported a variable and very high mass-loss rate, with values as high as $3 \times 10^{-3} M_{\odot} \mathrm{yr}^{-1}$ in the past, but having shown a sharp drop to a still considerable $\sim 4 \times 10^{-5} M_{\odot} \mathrm{yr}^{-1}$ about 1200 years ago.

AFGL 2343 exhibits the faintest set of submm lines of the sample studied here. In particular, it is the only source where no emission is detected in the ${ }^{12} \mathrm{CO} J=16-15$ and ${ }^{13} \mathrm{CO} J=10-9$ transitions. This is in line with the modelling results reported by CC07, where AFGL 2343 appears as a detached shell, with relatively low temperatures in general and low density in the central layers. A similar picture is derived from high resolution mid-IR maps (Lagadec et al. 2011). CC07 deduced a temperature of lower than about $20 \mathrm{~K}$ for the densest shell and between $\sim 300 \mathrm{~K}$ and $30 \mathrm{~K}$ for the much-less-dense inner component. These low temperatures may however be underestimated given the detection of several transitions with energy levels near $\sim 300 \mathrm{~K}$ (e.g. ${ }^{12} \mathrm{CO} J=10-9, o-\mathrm{H}_{2} \mathrm{O} 3_{2,1}-3_{1,2}$ or $\left.\mathrm{OH}\right)$. The 
lack of dense enough warm layers in this source is corroborated by the ${ }^{12} \mathrm{CO} J=10-9 / J=6-5$ line and integrated line ratios $(\sim 0.58$ and $\sim 0.5$, respectively). These values are significantly smaller than the corresponding line and integrated line ratios for IRC +10420 , which reach $\sim 0.77$ and $\sim 0.71$ respectively, confirming that most of the circumstellar gas in AFGL 2343 is cooler than the dominant circumstellar shells in IRC+10420.

The various line profiles have a red-shifted excess at $v_{\mathrm{LSR}} \sim$ $125 \mathrm{~km} \mathrm{~s}^{-1}$. The contrast between this narrow component and the emission around the star systemic velocity is higher for higher excitation lines. The same behaviour was observed in the mm-wave lines of several molecules (e.g. SiO, QuintanaLacaci et al. 2007). In contrast, this excess tends to disappear in lower excitation lines and is for example hardly seen in the ${ }^{12} \mathrm{CO} J=1-0$ and $J=2-1$ spectra (Bujarrabal et al. 2001). Interestingly, the ${ }^{12} \mathrm{CO} J=10-9 / J=6-5$ line ratio at this velocity increases to $\sim 0.81$, implying that there is a gas component in AFGL 2343 with excitation conditions at least comparable to those present in IRC +10420 .

The origin of this spectral component remains unclear. In the interferometric maps of ${ }^{12} \mathrm{CO} J=1-0$ and $J=2-1$ by $\mathrm{CC} 07$, this feature appears as a compact clump located in the same direction as the central star and moving almost at the extreme positive $L S R$ velocity. It would then correspond to a part of the envelope placed basically behind the star. Quintana-Lacaci et al. (2008) studied this component in more detail using maps of ${ }^{29} \mathrm{SiO} J=2-1$ and $\mathrm{HCN} J=1-0$ emission. They concluded that this feature comes from a component at relatively small radius of high density and temperature, and deduced it to be a very clumpy, thin shell. These authors suggest that the high-excitation condensations probably result from anisotropic shock propagation, particularly by analogy with the case of IRC +10420 , in which signs of shocks running within the envelope were also found from $\mathrm{SiO}$ emission maps.

We speculate that the spectral feature found at $125 \mathrm{~km} \mathrm{~s}^{-1}$ could also result from a shock between a hemisphere of the circumstellar shell and nearby interstellar gas. It is remarkable that the LSR velocity of AFGL 2343 is positive and high in absolute value, $\sim 100 \mathrm{~km} \mathrm{~s}^{-1}$, so the object is receding from us at high velocity. On the other hand, the low- $J$ CO observations (Bujarrabal et al. 2001) show that there is interstellar emission at moderate $L S R$ velocities, between $5 \mathrm{~km} \mathrm{~s}^{-1}$ and $10 \mathrm{~km} \mathrm{~s}^{-1}$. We cannot be sure whether this gas is really close to the YHG, but if this were the case, the strong difference in the projected velocities should produce a shock in the shell hemisphere placed beyond the star, which would show an $L S R$ velocity equal to approximately the stellar velocity plus the circumstellar expansion velocity, i.e. about $125 \mathrm{~km} \mathrm{~s}^{-1}$.

\section{Modelling of the $\mathrm{CO}$ emission in IRC+10420}

\subsection{The model}

CC07 developed a detailed model of the structure and kinematics of the CSE around IRC+10420 based on interferometric CO $J=$ $1-0$ and $J=2-1$ maps, together with the sub-mm observations of CO transitions up to $J=6-5$ performed by Teyssier et al. (2006).

We performed numerical calculations of $\mathrm{CO}$ line excitation and radiative transfer using a code very similar to that described in CC07. Despite the evidence of departure from spherical symmetry discussed in $\mathrm{CC} 07$ and Sect. 3.3, this model assumed isotropic mass loss, and therefore used a one-dimensional code. The line excitation and the population of the rovibrational

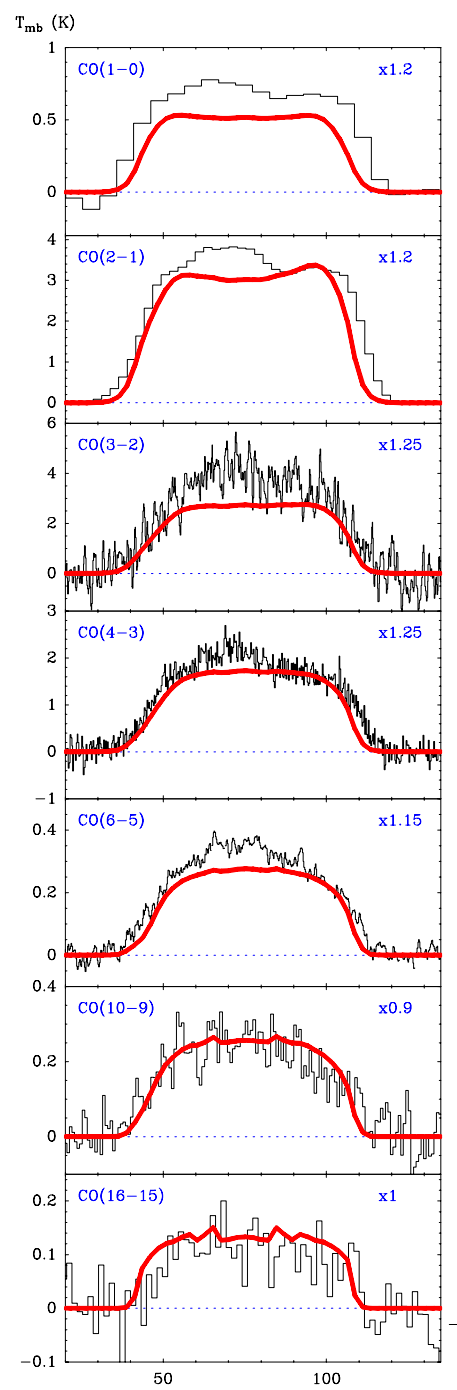

Velocity $(\mathrm{km} / \mathrm{s})$

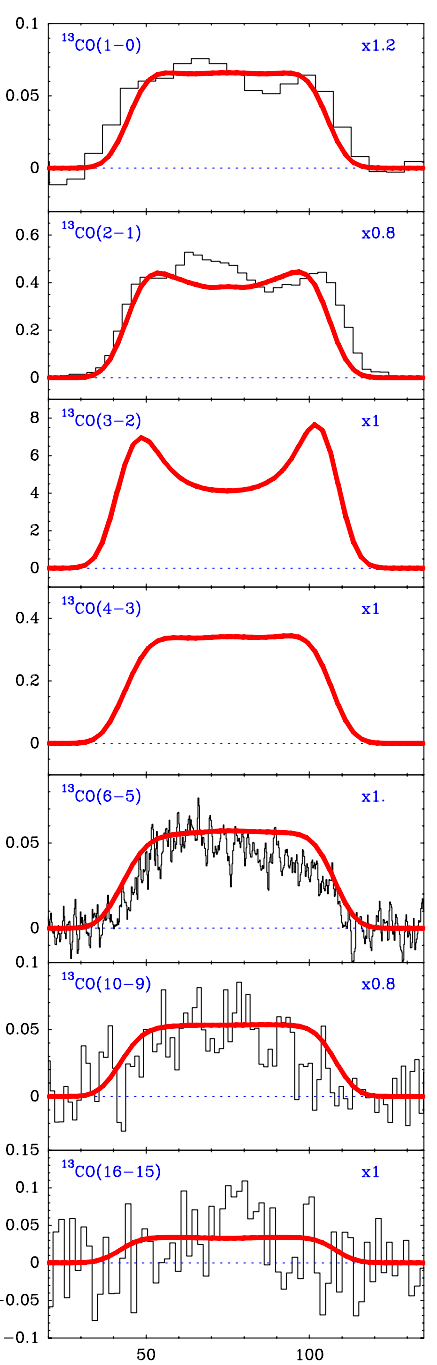

Velocity $(\mathrm{km} / \mathrm{s})$
Fig. 7. Results of the model for IRC+10420 (red line) overlaid on the line profiles presented in Fig. 5, as well as recent IRAM $30 \mathrm{~m}$ data of the $J=1-0$ and $J=2-1$ transitions from Quintana-Lacaci et al. (in prep.) and the ${ }^{12} \mathrm{CO} J=3-2$ and $J=4-3$ transitions from the JCMT archive. Note that no data were found for the $J=3-2$ and $J=4-3$ transitions of ${ }^{13} \mathrm{CO}$. For each line, the model was adjusted by a free corrective factor of at most the absolute-calibration accuracy error, which was applied to the given transition. For the JCMT archive data, this calibration accuracy was unclear to us so we used a conservative upper limit of the $25 \%$ error. For the IRAM 30 m data, calibration errors of $20 \%$ were assumed at both $3 \mathrm{~mm}$ and $1 \mathrm{~mm}$. The correction factor is indicated in the upper right corner of each box.

levels were calculated using an large-velocity-gradient (LVG) approach, which is justified in view of the large macroscopic velocities found in this object. The brightness distribution for each line was calculated by solving the "exact" radiative transfer equations, so radiative interaction between distant points and opacity effects were fully taken into account. The brightness distribution was later convolved with the corresponding telescope beams, which were assumed to be Gaussian.

The original model by CCO7 consisted of two detached shells, each one corresponding to large mass loss episodes spaced at interval of 1200 years. It also assumed that the mass loss ceased 200 years ago. These respective mass ejections expand at high velocities $\left(37 \mathrm{~km} \mathrm{~s}^{-1}\right.$ and $25 \mathrm{~km} \mathrm{~s}^{-1}$ for the inner 
and outer shells, respectively), as expected given the high luminosity of these objects. Following $\mathrm{CC} 07$, the inner shell is assumed to be located between $2.5 \times 10^{16} \mathrm{~cm}$ and $1.2 \times 10^{17} \mathrm{~cm}$ and have a mass-loss rate of $3 \times 10^{-4} M_{\odot} \mathrm{yr}^{-1}$. The outer shell is located between $2.2 \times 10^{17} \mathrm{~cm}$ and $5.2 \times 10^{17} \mathrm{~cm}$ and has a rate of $1.2 \times 10^{-4} M_{\odot} \mathrm{yr}^{-1}$. The kinetic temperatures were described by a power law $T_{\mathrm{k}}(r)=T_{17}\left(r / 10^{17} \mathrm{~cm}\right)^{\alpha}$, where $T_{17}$ is the temperature at $10^{17} \mathrm{~cm}$ from the star. The gas temperatures derived by CC07 were high, $T_{17}=230 \mathrm{~K}$ and $T_{17}=100 \mathrm{~K}$ for the inner and outer shells, respectively. Values of $\alpha \sim-1$ were found. However, we note that low- $J$ transitions are not good tracers of high-excitation regions, so that HIFI observations are necessary to accurately constrain the temperature.

\subsection{Model results: comparison with Herschel/HIFI data}

We compared the outcome of high- $J$ line profiles predicted by this CSE model with our Herschel/HIFI data. Although that model was derived from data of low-excitation lines, we find that it can fit all the high- $J$ CO lines observed by HIFI by simply lowering the kinetic temperature for the inner shell from $T_{17}=230 \mathrm{~K}$ to $T_{17}=170 \mathrm{~K}$ in order to fit the ${ }^{12} \mathrm{CO} J=16-15$ line. This resulted in an average kinetic temperature of $\sim 700 \mathrm{~K}$ in the innermost part of the CSE. We also note that the high- $J$ line intensities are fully reproducible by restricting the model to an outer radius of $1.1 \times 10^{17} \mathrm{~cm}$, i.e. roughly the inner shell observed by $\mathrm{CC} 07$. This indicates that the outer shell around IRC+10420 is not probed by the Herschel/HIFI $\mathrm{CO}$ observations.

The result of the fitting of the HIFI ${ }^{12} \mathrm{CO}$ and ${ }^{13} \mathrm{CO}$ lines up to $J=16-15$ from IRC +10420 is shown in Fig. 7 . The corresponding mass-loss and kinetic temperature profiles are displayed in Fig. 8. We found that two different temperature distributions can reproduce the data (Fig. 8). In the first case, we adopted the same (relatively steep) slope $\alpha=-1.2$ as CC07, which led to a global decrease in the temperature to $T_{17}=170 \mathrm{~K}$. However, we were also able to reproduce the observed lines we we considered a more gentle increase in the temperature toward the centre, of $\alpha=-0.8$ and kept the original value of $T_{17}=230 \mathrm{~K}$. In both models, the highest temperature of the circumstellar gas around IRC+10420 remains $\$ 700 \mathrm{~K}$, instead of the values $\gtrsim 1000 \mathrm{~K}$ originally assumed by CC07 (see Fig. 8).

The assumed ${ }^{12} \mathrm{CO}$ abundance was $3 \times 10^{-4}$ (typical of O-rich envelopes), and the ${ }^{13} \mathrm{CO}$ abundance was taken from the study of Quintana-Lacaci et al. (2007). This translates into a ${ }^{12} \mathrm{CO} /{ }^{13} \mathrm{CO}$ abundance ratio of order $1 / 5$, which is relatively high compared to AGBs but very close to that reported in highmass post-red supergiants (see CC07, Quintana-Lacaci et al. 2007).

\section{Conclusion}

We have reported Herschel/HIFI observations of high spectral resolution for FIR/submm molecular lines in two red supergiant stars (RSGs), NML Cyg and Betelgeuse, and two yellow hypergiant stars (YHGs), IRC+10420 and AFGL 2343. Tables 3 and 4 summarize the observational parameters derived from our data, which are displayed in Figs. 2 to $6-$ the full spectra are shown in the Appendix.

As illustrated in Fig. 9, the RSGs exhibit in general more intense high-excitation lines, indicating that they harbour inner circumstellar layers with high temperatures close to or even higher than $1000 \mathrm{~K}$ (Sects. 3.1 and 3.2). In contrast, YHGs do not

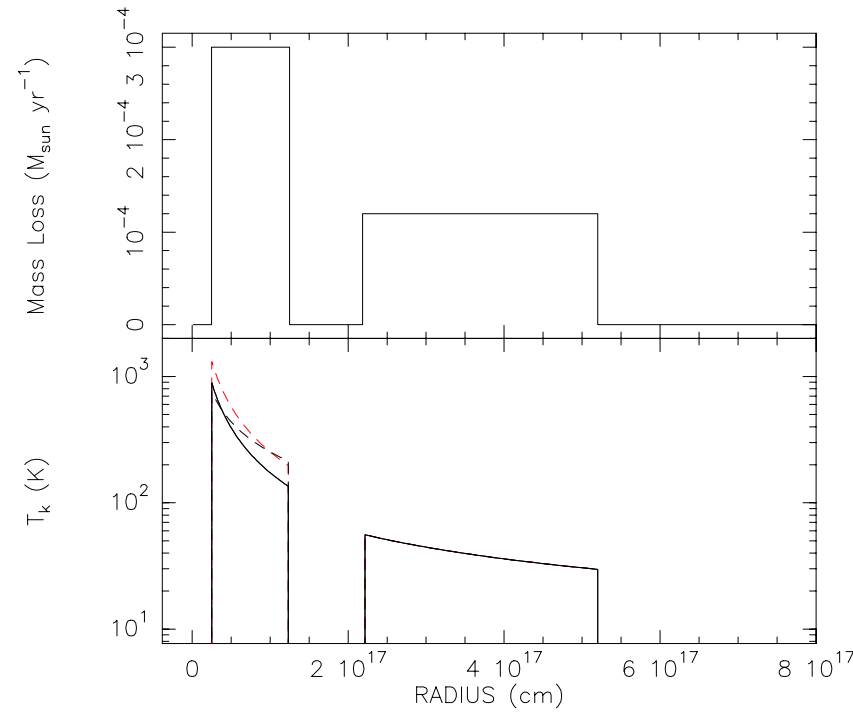

Fig. 8. Upper panel: mass-loss pattern as a function of stellar radius derived for the model of IRC+10420 (Sect. 4.2). Lower panel: kinetic temperature profile as a function of stellar radius for the model of IRC +10420 . The solid line corresponds to $T_{17}=170 \mathrm{~K}$ and $\alpha=-1.2$, while the black dashed line uses $T_{17}=230 \mathrm{~K}$ and $\alpha=-0.8$. The red dashed line illustrates the inner layer profile assumed in the model of $\operatorname{CC07}\left(T_{17}=230 \mathrm{~K}\right.$ and $\left.\alpha=-1.2\right)$.

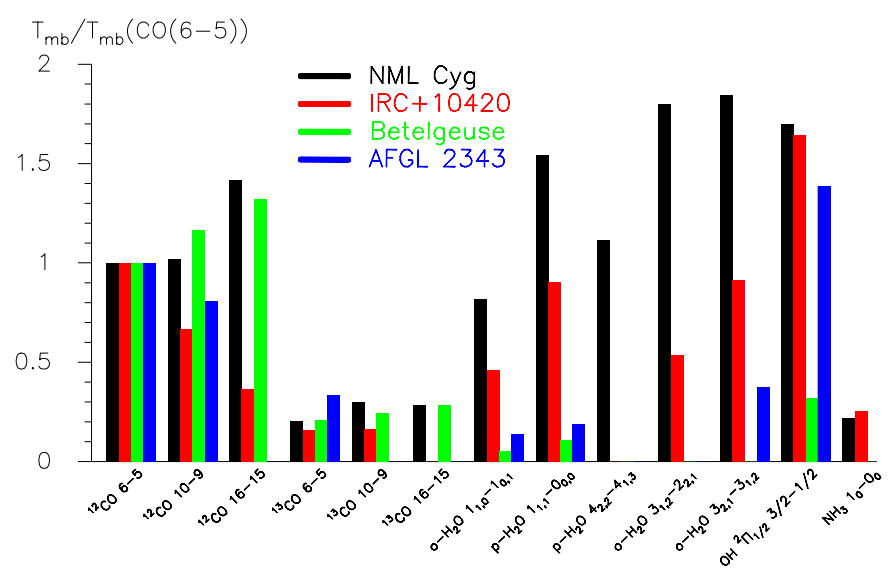

Fig. 9. Histograms of the peak brightness temperatures as tabulated in Tables 3 and 4, for lines detected in at least 2 sources, normalised by the ${ }^{12} \mathrm{CO} J=6-5$ intensity of the respective sources. Non-detected lines are not represented in this graph.

contain this central warm material, and are instead surrounded by detached envelopes with low densities at small distances from the star (Sects. 3.3 and 3.4). As such, our results show that the mass-loss rates in YHGs are presently weak. We also observe that the spectral line profiles in RSGs tend to become narrower with increasing excitation temperature. We show that this phenomenon indicates that the high excitation lines in RSGs originate from gas layers at small radii that have not yet reached the circumstellar terminal velocity. This trend is not observed in YHGs, in line with the picture of a hollow envelope closer to the stellar photosphere. These YHGs are thus less likely than RSGs to contain gas still in the acceleration phase.

In both YHGs, we detected intense emission features that are conspicuous in the profiles. That of IRC +10420 is relatively blue-shifted, at $v_{\mathrm{LSR}} \sim 65 \mathrm{~km} \mathrm{~s}^{-1}$, and is more noticeable in certain molecules, such as $\mathrm{H}_{2} \mathrm{O}$ and $\mathrm{NH}_{3}$, although it does not seem to require particular excitation conditions. It was detected 
and mapped in low- $J$ CO Lines by CC07, who concluded that it comes from a condensation in the outer envelope. We also found a peculiar emission excess in AFGL 2343 (Sect. 3.4), at an extreme positive velocity of $v_{\mathrm{LSR}} \sim 125 \mathrm{~km} \mathrm{~s}^{-1}$. There, the feature is clearly associated with the excitation of the lines. We speculate that this shell could result from a shock interaction between the circumstellar envelope and nearby interestellar gas.

We stress the particularly strong emission of water lines in NML Cyg and IRC+10420, which are also the only two sources featuring $\mathrm{NH}_{3}$ emission. On the other hand, $\mathrm{OH}$ is ubiquitously observed. It is unclear where this molecule predominantly arises and how it forms in the envelopes. Its emission does not seem to be correlated with that of $\mathrm{H}_{2} \mathrm{O}$, although $\mathrm{OH}$ formation in evolved stars is thought to result from $\mathrm{H}_{2} \mathrm{O}$ photodissociation; for instance, the observed $\mathrm{OH}$ profiles are usually closer to those of mid-excitation $\mathrm{CO}$ lines and the $\mathrm{OH}$ line intensity is high in AFGL 2343, which shows particularly weak water lines.

Finally, we have proposed a preliminary model to fit the ${ }^{12} \mathrm{CO}$ and ${ }^{13} \mathrm{CO}$ lines detected in IRC +10420 . The model is based on that developed by $\mathrm{CC} 07$ to explain their mm-wave interferometric mapping. We found that the original description of the shell around IRC +10420 by $\mathrm{CC} 07$ is able to reproduce all transitions by simply decreasing the temperature of the inner layers by about $30 \%$ (Fig. 8). In addition, the emission from the high- $J$ lines is found to originate only from a detached, hot shell close to the star, formed by stellar mass ejection at a high rate of $\sim 3 \times 10^{-4} M_{\odot} \mathrm{yr}^{-1}$. Our calculation indicates that no hotter components at small radii are needed to reproduce the molecular emission of IRC +10420 , confirming that the heavy mass-loss ceased about 200 years ago. More detailed modelling of the $\mathrm{CO}$ lines observed in IRC+10420 and AFGL 2343 will be presented in a forthcoming paper, in particular to take into account the departure from spherical symmetry illustrated by high-resolution maps at various wavelengths, and corroborated by the distinct spectral features detected in the HIFI data.

Acknowledgements. The authors would like to thank the anonymous referee for constructive comments, allowing in particular this paper to become more concise. HIFI has been designed and built by a consortium of institutes and university departments from across Europe, Canada, and the United States under the leadership of SRON Netherlands Institute for Space Research, Groningen, The Netherlands and with major contributions from Germany, France, and the US. Consortium members are: Canada, CSA, U. Waterloo; France, CESR, LAB, LERMA, IRAM; Germany, KOSMA, MPIfR, MPS; Ireland, NUI Maynooth; Italy, ASI, IFSI-INAF, Osservatorio Astrofisico di Arcetri - INAF; The Netherlands, SRON, TUD; Poland, CAMK, CBK; Spain, Observatorio Astronómico Nacional (IGN), Centro de Astrobiología (CSIC-INTA); Sweden,
Chalmers University of Technology - MC2, RSS \& GARD, Onsala Space Observatory, Swedish National Space Board, Stockholm University - Stockholm Observatory; Switzerland, ETH Zurich, FHNW; USA, Caltech, JPL, NHSC. This work was supported by the German Deutsche Forschungsgemeinschaft, $D F G$ project number Os $177 / 1-1$. A portion of this research was performed at the Jet Propulsion Laboratory, California Institute of Technology, under contract with the National Aeronautics and Space Administration. RSz and MSch ackowledge support from grant N203 581040 of National Science Center.

\section{References}

Boboltz, D. A., \& Marvel, K. B. 2000, ApJ, 545, L149

Bujarrabal, V., Castro-Carrizo, A., Alcolea, J., et al. 2001, A\&A, 377, 868

Bujarrabal, V., Alcolea, J., Soria-Ruiz, R., et al. 2012, A\&A, 537, A8

Castro-Carrizo, A., Bujarrabal, V., Fong, D., et al. 2001, A\&A, 367, 674

Castro-Carrizo, A., Quintana-Lacaci, G., Bujarrabal, V., et al. 2007, A\&A, 465, 457

De Beck, E., Decin, L., de Koter, A., et al. 2010, A\&A, 523, A18

Decin, L., Hony, S., de Koter, A., et al. 2006, A\&A, 456, 549

de Graauw, T., Helmich, F. P., Phillips, T. G., et al. 2010, A\&A, 518, L4

de Jager, C. 1998, A\&ARv, 8, 145

Ferguson, B. A., \& Ueta, T. 2010, ApJ, 711, 613

Gledhill, T. M., Yates, J. A., \& Richards, A. M. S. 2001, MNRAS, 328, 301

Haas, M. R., \& Glassgold, A. E. 1993, ApJ, 410, L111

Hawkins, G. W., Skinner, C. J., Meixner, M. M., et al. 1995, ApJ, 452, 314

Josselin, E., \& Plez, B. 2007, A\&A, 469, 671

Justtanont, K., Tielens, A. G. G. M., de Jong, T., et al. 1999, A\&A, 345, 605

Justtanont, K., Khouri, T., Maercker, M., et al. 2012, A\&A, 537, A144

Kemper, F., Starck, R., Justtanont, K., et al. 2003, A\&A, 407, 609

Kervella, P., Perrin, G., Chiavassa, A., et al. 2011, A\&A, 531, A117

Lagadec, E., Verhoelst, T., Mékarnia, D., et al. 2011, MNRAS, 417, 32

Levesque, E. M. 2010, Hot and Cool: Bridging Gaps in Massive Star Evolution, 425,103

Lis, D. C., Phillips, T. G., Goldsmith, P. F., et al. 2010, A\&A, 521, L26

Mauron, N., \& Josselin, E. 2011, A\&A, 526, A156

Menten, K. M., \& Young, K. 1995, ApJ, 450, L67

Menten, K. M., Wyrowski, F., Alcolea, J., et al. 2010, A\&A, 521, L7

Meynet, G., \& Maeder, A. 2003, A\&A, 404, 975

Monnier, J. D., Millan-Gabet, R., Tuthill, P. G., et al. 2004, ApJ, 605, 436

Nagayama, T., Takeda, K., Omodaka, T., et al. 2008, PASJ, 60, 1069

Pilbratt, G. L., Riedinger, J. R., Passvogel, T., et al. 2010, A\&A, 518, L1

Quintana-Lacaci, G., Bujarrabal, V., Castro-Carrizo, A., \& Alcolea, J. 2007, A\&A, 471, 551

Quintana-Lacaci, G., Bujarrabal, V., \& Castro-Carrizo, A. 2008, A\&A, 488, 203

Reddy, B. E., \& Hrivnak, B. J. 1999, AJ, 117, 1834

Rodgers, B., \& Glassgold, A. E. 1991, ApJ, 382, 606

Roelfsema, P., Helmich, F. P., Teyssier, D., et al. 2012, A\&A, 537, A17

Rygl, K. L. J., Brunthaler, A., Sanna, A., et al. 2012, A\&A, 539, A79

Schuster, M. T., Marengo, M., Hora, J. L., et al. 2009, ApJ, 699, 1423

Tenenbaum, E. D., Dodd, J. L., Milam, S. N., Woolf, N. J., \& Ziurys, L. M. 2010, ApJS, 190, 348

Teyssier, D., Hernandez, R., Bujarrabal, V., et al. 2006, A\&A, 450, 167

Zhang, B., Reid, M. J., Menten, K., et al. 2012, A\&A, 544, A42 
D. Teyssier et al.: Herschel/HIFI observations of red supergiants and yellow hypergiants. I.

\section{Appendix A: Full HIFI spectra}

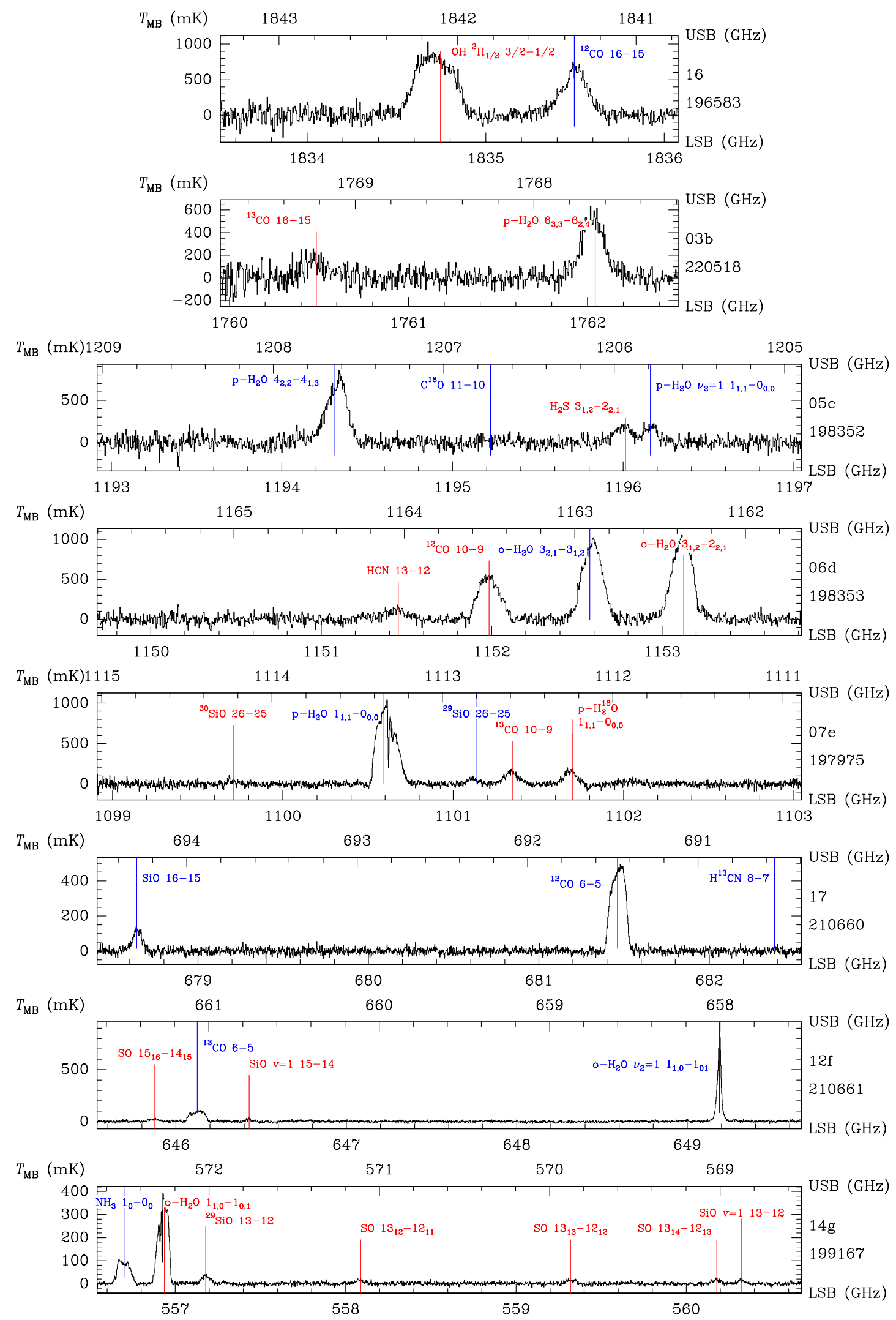

Fig. A.1. Full wide-band spectrometer spectra obtained towards NML Cyg. The setting number is indicated on the right side. Each spectrum is displayed on a double-side-band scale with both side-band frequency scales drawn. The spectral resolution is the native one (1.1 MHz), and loworder polynomial baselines have been subtracted. Lines are indicated with the respective ticks at the expected (velocity-corrected) frequency lines from the USB have their ticks pointing upwards, while lines from the LSB have ticks pointing downwards. The spectrum in setting 16 is made of only a fraction of the data taken at this frequency, for the purpose of standing wave mitigation (see text for details). The obsid's numbers are given below the setting number, in the form of $1342 \times$, " $\times$ " being the identifier indicated on the plot. 


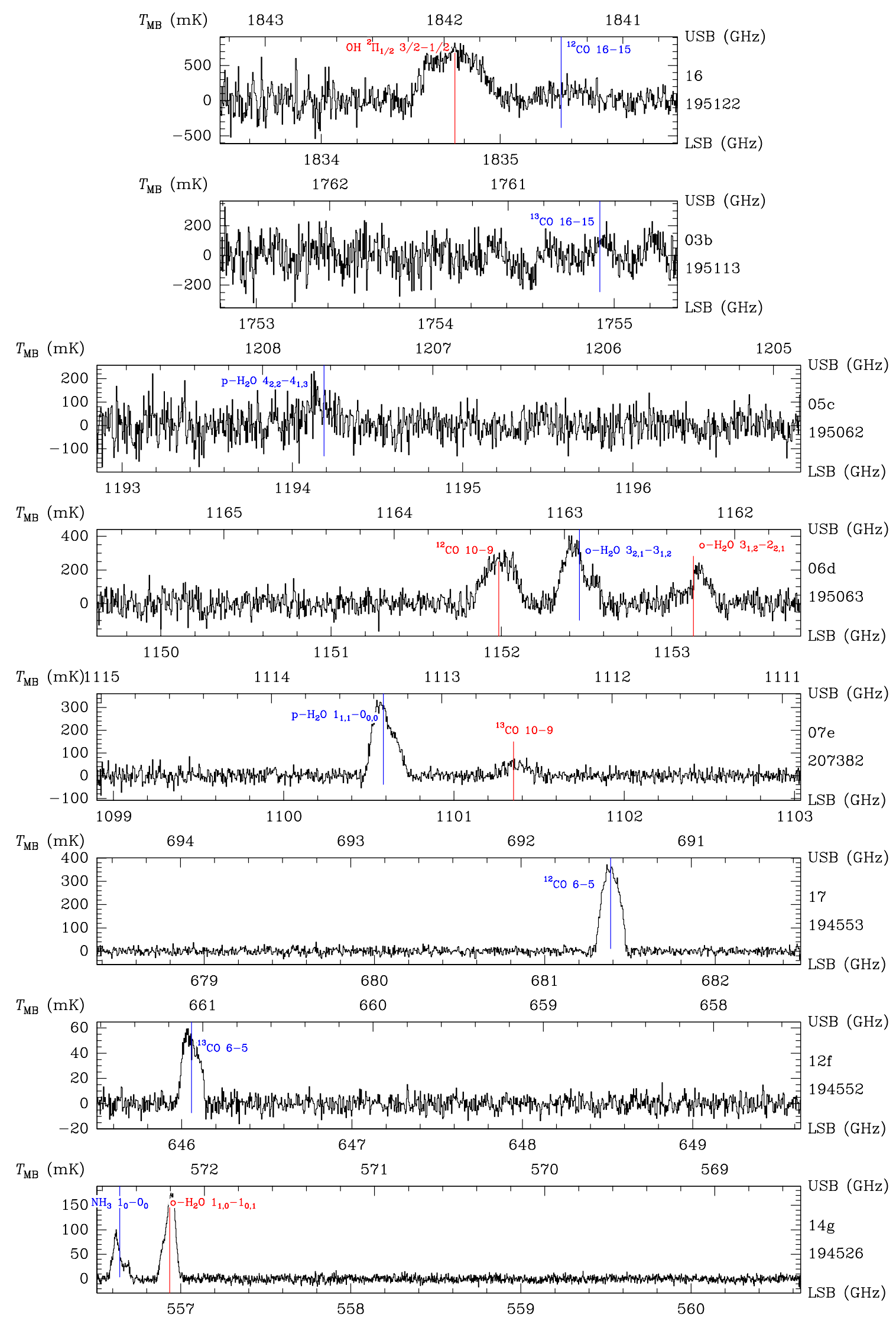

Fig. A.2. Same as Fig. A.1 for IRC+10420. The spectrum in setting $16 \times$ is made of only a fraction of the data taken at this frequency, for the purpose of standing wave mitigation (see text for details). The tick displayed for ${ }^{13} \mathrm{CO} J=16-15$ illustrates the expected position of this line, despite its non-detection. 
D. Teyssier et al.: Herschel/HIFI observations of red supergiants and yellow hypergiants. I.
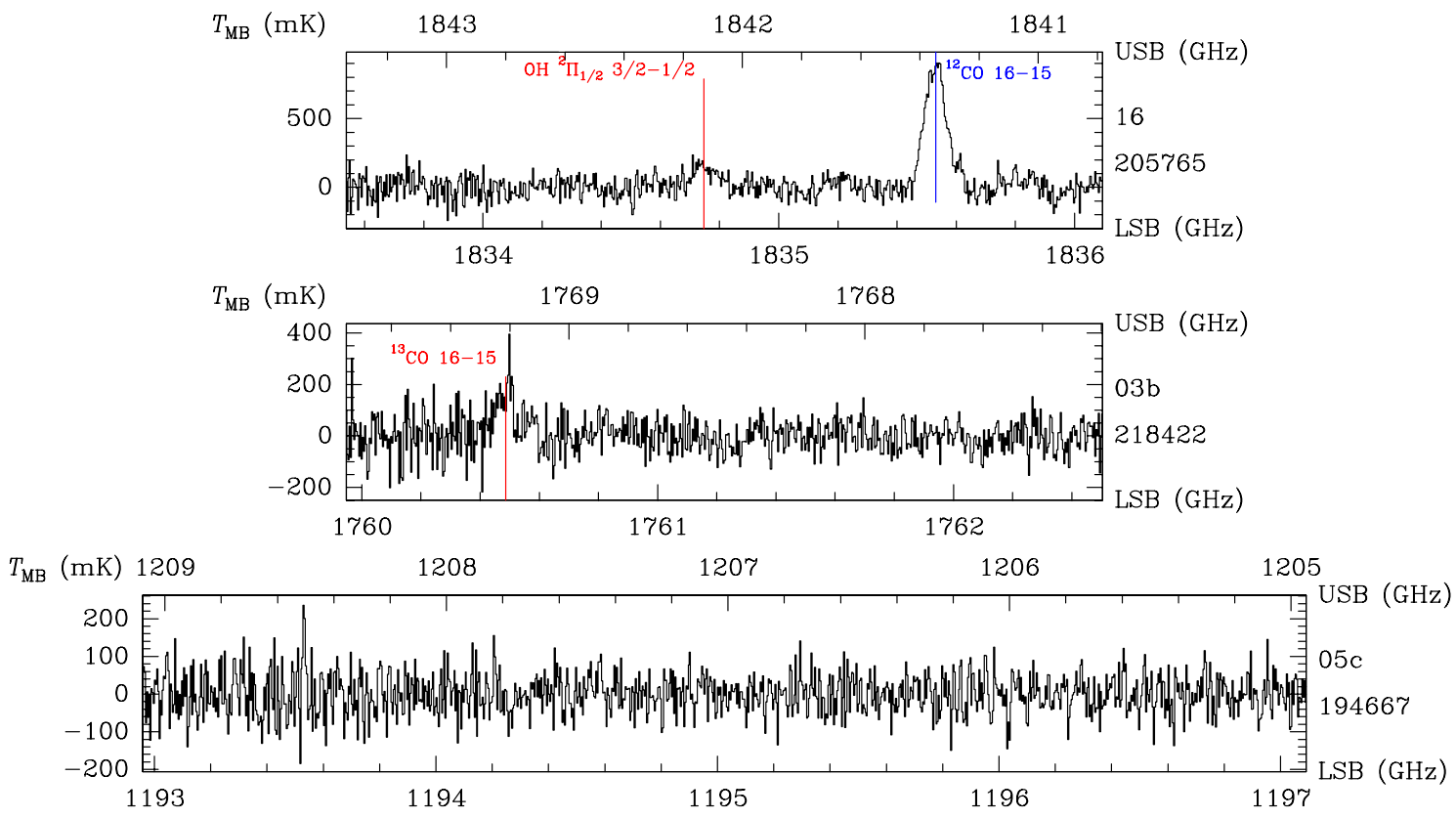

$\begin{array}{lllll}T_{\text {MB }}(\mathrm{mK}) & 1165 & 1164 & 1163 & 1162\end{array}$
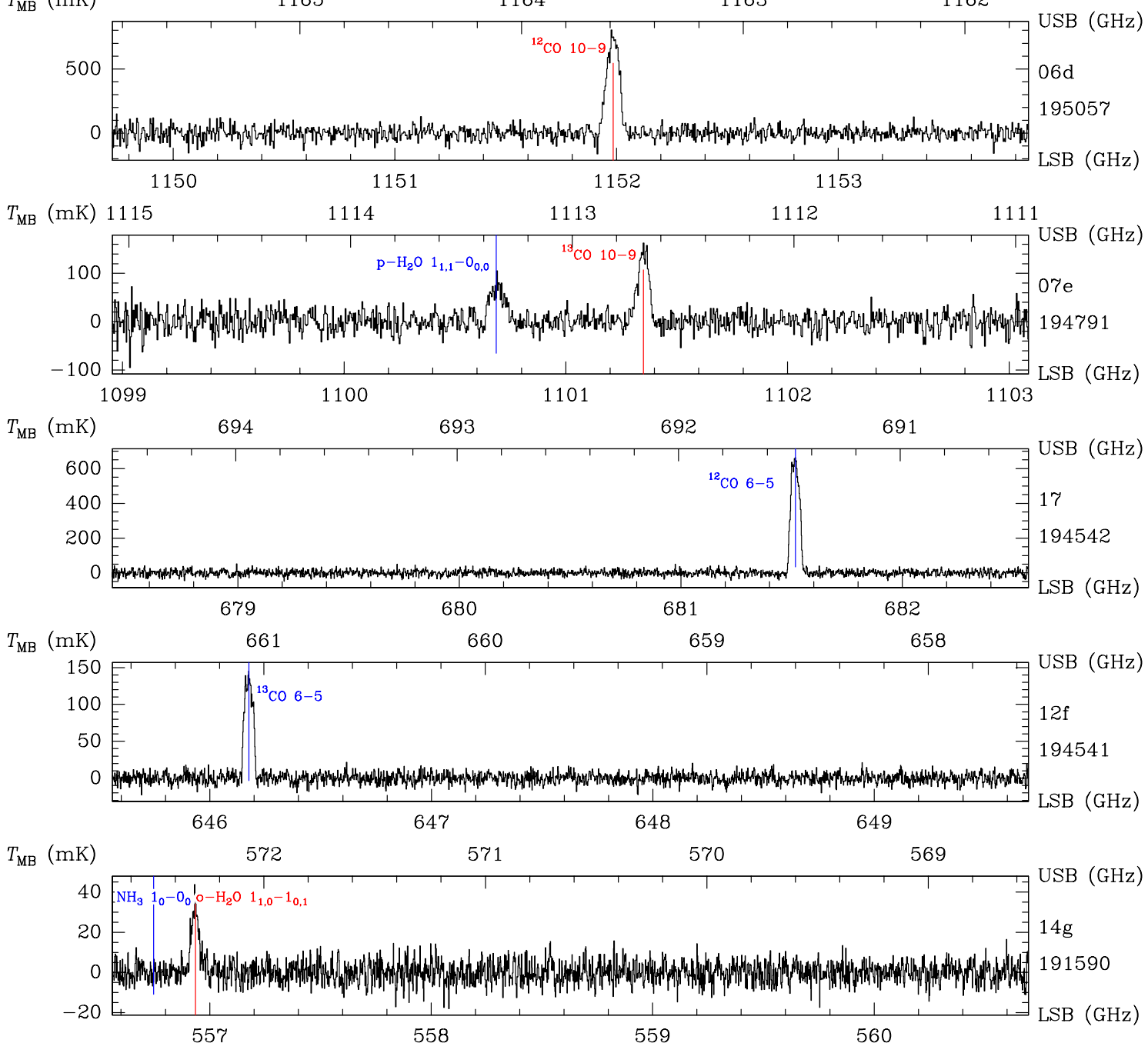

Fig. A.3. Same as Fig. A.1 for Betelgeuse. The tick displayed for $\mathrm{NH}_{3}$ illustrates the expected position of this line, despite its non-detection. 

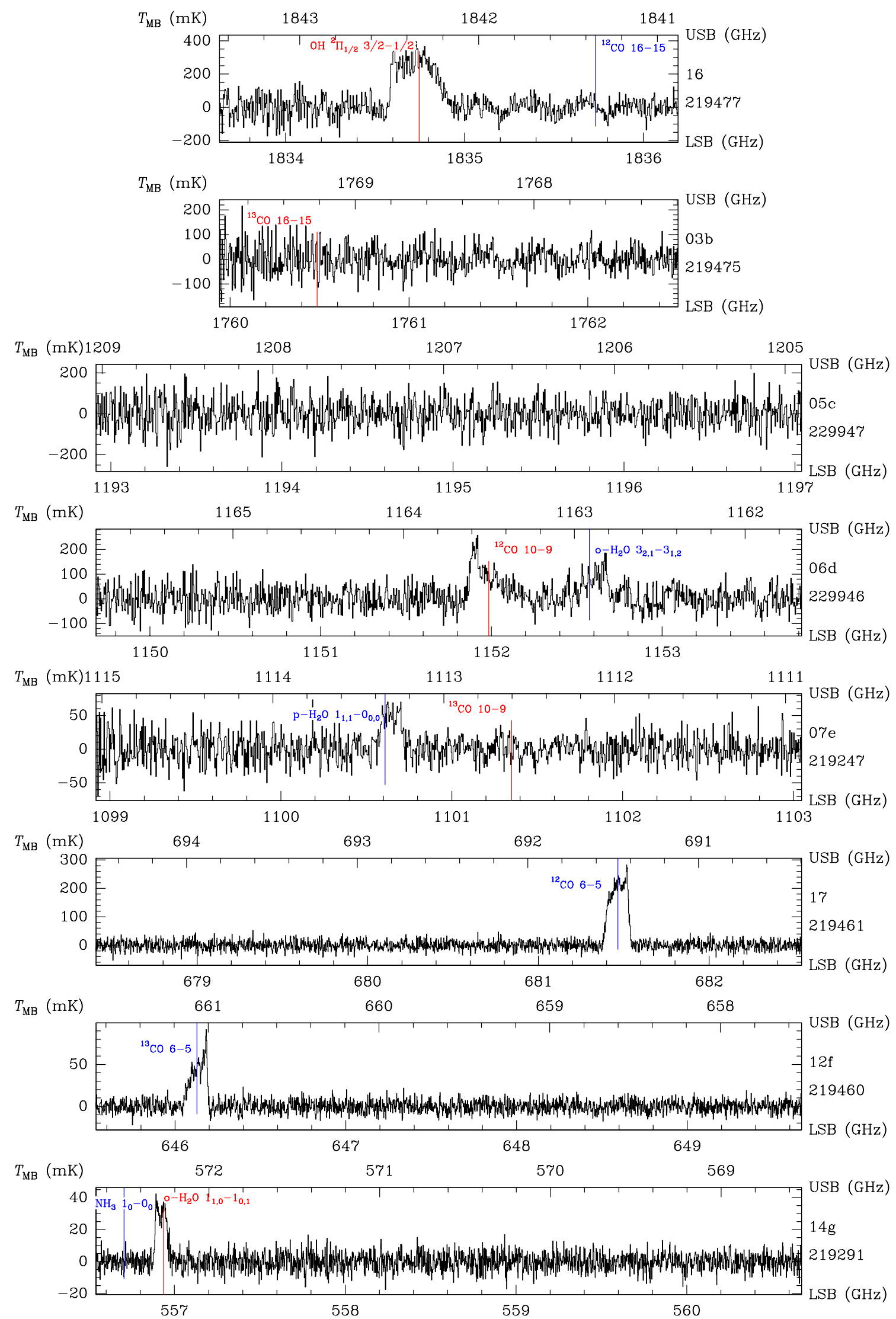

Fig. A.4. Same as Fig. A.1 for AFGL 2343. The ticks displayed for $\mathrm{NH}_{3},{ }^{13} \mathrm{CO} J=10-9,{ }^{12} \mathrm{CO} J=16-15$ and ${ }^{13} \mathrm{CO} J=16-15$ illustrate the expected position of these lines, despite their non-detection. 